\title{
A Stabilized Finite Element Method for the Darcy Problem on Surfaces *
}

\author{
Peter Hansbo ${ }^{\dagger}$ Mats G. Larson ${ }^{\ddagger}$
}

March 26, 2022

\begin{abstract}
We consider a stabilized finite element method for the Darcy problem on a surface based on the Masud-Hughes formulation. A special feature of the method is that the tangential condition of the velocity field is weakly enforced through the bilinear form and that standard parametric continuous polynomial spaces on triangulations can be used. We prove optimal order a priori estimates that take the approximation of the geometry and the solution into account.
\end{abstract}

\section{Introduction}

In this note we develop a stabilized finite element method for Darcy flow on triangulations of a smooth surface. Starting from the Masud-Hughes formulation [12] we obtain a very convenient method based on a coercive bilinear form that can handle different approximation spaces. More precisely, we consider parametric continuous piecewise polynomial elements, with possibly different orders in the mapping as well as the spaces for the velocity and pressure. A special feature of our approach is that we avoid using vector elements and discretize the tangent velocity vector componentwise in $\mathbb{R}^{3}$ together with a weak enforcement of the tangent condition. Our approach is in contrast with the recent report [7] where a method for Darcy flow based on Raviart-Thomas spaces was presented.

We derive error estimates that takes the approximation of the geometry and the solution into account and separates the dependency of the different orders of approximations. The error in the velocity is defined using standard componentwise liftings based on the closest

*This research was supported in part by the Swedish Foundation for Strategic Research Grant No. AM13-0029, the Swedish Research Council Grants No. 2011-4992 and No. 2013-4708, and Swedish strategic research programme eSSENCE.

†Department of Mechanical Engineering, Jönköping University, SE-551 11 Jönköping, Sweden, Peter.Hansbo@jth.hj.se

${ }^{\ddagger}$ Department of Mathematics and Mathematical Statistics, Umeå University, SE-901 87 Umeå, Sweden, mats.larson@math.umu.se 
point mapping and we show an energy estimate, an $L^{2}$ estimate for the pressure, and an $L^{2}$ estimate for the tangential part of the velocity which is slightly sharper with respect to the geometry approximation compared to the bound for the full velocity vector provided by the energy norm estimate. We also provide numerical results confirming our theoretical investigations.

Recently there has been an increasing activity in research on finite elements on surfaces, in particular, we mention the following references that are relevant to this work: finite element methods for membrane shell problem based on tangential calculus [9] (linear) and [10] (nonlinear), higher order methods for the Laplace-Beltrami operator [4], continuousdiscontinuous Galerkin methods for the biharmonic problem [11], and the seminal paper [5] where finite elements for the Laplace-Beltrami was first developed. For general background on finite elements for PDEs on surfaces we refer to the recent review article [6] and the references therein.

The outline of the reminder of the paper is as follows: In Section 2 we precent the Darcy problem on a surface and the necessary background on tangential calculus. In Section 3 we define the triangulations and their approximation properties, the finite element spaces, the interpolation theory, and finally the finite element method. In Section 4 we collect necessary results on lifting and extension of functions between the exact and discrete surfaces. In Section 5 we derive a priori error estimates starting with a Strang lemma and then estimates of the quadrature errors in the forms resulting approximation of the geometry, which together with the interpolation results yields the final estimate. Estimates for the presssure and tangential part of the velocity are derived using duality techniques. Finally, in Section 6 we present numerical examples.

\section{The Darcy Problem on a Surface}

\subsection{The Surface}

Let $\Gamma$ be a closed smooth surface embedded in $\mathbb{R}^{3}$ with signed distance function $\rho$, exterior unit normal $\boldsymbol{n}=\nabla \rho$, and closest point mapping $\boldsymbol{p}: \mathbb{R}^{3} \rightarrow \Gamma$. Then there is a $\delta_{0}>$ such that $\boldsymbol{p}$ maps each point in $U_{\delta_{0}}(\Gamma)$ to precisely one point on $\Gamma$, where $U_{\delta}(\Gamma)=\left\{\boldsymbol{x} \in \mathbb{R}^{3}: \rho(\boldsymbol{x})<\delta\right\}$ is an open tubular neighborhood of $\Gamma$.

\subsection{Tangential Calculus}

For each function $u$ defined on $\Gamma$ we let the extension $u^{e}$ to the neighborhood $U_{\delta_{0}}(\Gamma)$ be defined by the pull back $u^{e}=u \circ \boldsymbol{p}$. For a function $u: \Gamma \rightarrow \mathbb{R}$ we then define the tangential gradient

$$
\nabla_{\Gamma} u=\boldsymbol{P}_{\Gamma} \nabla u^{e}
$$

where $\boldsymbol{P}_{\Gamma}=\boldsymbol{I}-\boldsymbol{n} \otimes \boldsymbol{n}$ is the projection onto the tangent plane $T_{\boldsymbol{x}}(\Gamma)$. The surface divergence of a vector field $\boldsymbol{u}: \Gamma \rightarrow \mathbb{R}^{3}$ is defined by

$$
\operatorname{div}_{\Gamma}(\boldsymbol{u})=\operatorname{tr}\left(\boldsymbol{u} \otimes \nabla_{\Gamma}\right)=\operatorname{div}(\boldsymbol{u})-\boldsymbol{n} \cdot(\boldsymbol{u} \otimes \nabla) \cdot \boldsymbol{n}
$$


Decomposing $\boldsymbol{u}$ into a tangent and normal component

$$
\boldsymbol{u}=\boldsymbol{u}_{t}+u_{n} \boldsymbol{n}
$$

we have the identity

$$
\operatorname{div}_{\Gamma} \boldsymbol{u}=\operatorname{div}_{\Gamma} \boldsymbol{u}_{t}+u_{n} H
$$

where

$$
H=\operatorname{tr}\left(\boldsymbol{\kappa}_{\Gamma}\right)
$$

is twice the mean curvature of the surface and $\boldsymbol{\kappa}_{\Gamma}=\left.\boldsymbol{\kappa}\right|_{\Gamma}$, with $\boldsymbol{\kappa}=\nabla \otimes \nabla \rho$, is the curvature tensor of $\Gamma$. Using Green's formula we have

$$
\left(\operatorname{div}_{\Gamma} \boldsymbol{v}_{t}, q\right)_{\Gamma}=-\left(\boldsymbol{v}_{t}, \nabla_{\Gamma} q\right)_{\Gamma}
$$

for tangential vector fields $\boldsymbol{v}_{t}$.

\subsection{The Surface Darcy Problem}

Tangential Vector Field Formulation. The Darcy problem takes the form: find a tangential vector field $\boldsymbol{u}_{t}: \Gamma \rightarrow T(\Gamma)$ representing velocity and the pressure $p: \Gamma \rightarrow \mathbb{R}$ such that

$$
\begin{aligned}
\operatorname{div}_{\Gamma} \boldsymbol{u}_{t} & =f & & \text { on } \Gamma \\
\boldsymbol{u}_{t}+\nabla_{\Gamma} p & =\boldsymbol{g} & & \text { on } \Gamma
\end{aligned}
$$

where $f: \Gamma \rightarrow \mathbb{R}$ is a given function such that $\int_{\Gamma} f=0$ and $\boldsymbol{g}: \Gamma \rightarrow \mathbb{R}^{3}$ is a given tangential vector field. The corresponding weak form reads: find $\left(\boldsymbol{u}_{t}, p\right) \in \boldsymbol{V}_{t} \times Q$ such that

$$
a_{t}\left(\left(\boldsymbol{u}_{t}, p\right),\left(\boldsymbol{v}_{t}, q\right)\right)=l(q) \quad \forall\left(\boldsymbol{v}_{t}, q\right) \in \boldsymbol{V}_{t} \times Q
$$

where

$$
\begin{aligned}
a_{t}\left(\left(\boldsymbol{u}_{t}, p\right),\left(\boldsymbol{v}_{t}, q\right)\right) & =\left(\boldsymbol{u}_{t}, \boldsymbol{v}_{t}\right)_{\Gamma}+\left(\nabla_{\Gamma} p, \boldsymbol{v}_{t}\right)_{\Gamma}-\left(\boldsymbol{u}_{t}, \nabla_{\Gamma} q\right)_{\Gamma} \\
l(q) & =(f, q)_{\Gamma}+(\boldsymbol{g}, \boldsymbol{v})_{\Gamma}
\end{aligned}
$$

with $\boldsymbol{V}_{t}=\left\{\boldsymbol{v}: \Gamma \rightarrow \mathbb{R}^{3}: \boldsymbol{v} \in\left[L^{2}(\Gamma)\right]^{3}, \boldsymbol{n} \cdot \boldsymbol{v}=0\right\}$ and $Q=\left\{q \in H^{1}(\Gamma): \int_{\Gamma} q=0\right\}$.

Since $\Gamma$ is smooth and $p \in Q$ is the solution to the elliptic problem $\operatorname{div}_{\Gamma}\left(\nabla_{\Gamma} p\right)=$ $\operatorname{div}_{\Gamma} \boldsymbol{u}_{t}-\operatorname{div}_{\Gamma} \boldsymbol{g}=f-\operatorname{div}_{\Gamma} \boldsymbol{g}$, we have the elliptic regularity estimate

$$
\|p\|_{H^{s+2}(\Gamma)} \lesssim\left\|f-\operatorname{div}_{\Gamma} \boldsymbol{g}\right\|_{H^{s}(\Gamma)} \lesssim\|f\|_{H^{s}(\Gamma)}+\|\boldsymbol{g}\|_{H^{s+1}(\Gamma)}
$$

which combined with $\left\|\boldsymbol{u}_{t}\right\|_{H^{s+1}(\Gamma)}=\left\|\boldsymbol{g}-\nabla_{\Gamma} p\right\|_{H^{s+1}(\Gamma)} \leq\|\boldsymbol{g}\|_{H^{s+1}(\Gamma)}+\|p\|_{H^{s+2}(\Gamma)}$ gives

$$
\left\|\boldsymbol{u}_{t}\right\|_{H^{s+1}(\Gamma)}+\|p\|_{H^{s+2}(\Gamma)} \lesssim\|f\|_{H^{s}(\Gamma)}+\|\boldsymbol{g}\|_{H^{s+1}(\Gamma)}
$$


General Vector Field Formulation. Letting $\boldsymbol{u}$ be a vector field with a nonzero normal component and recalling the split $\boldsymbol{u}=\boldsymbol{u}_{t}+u_{n} \boldsymbol{n}$, see equation (2.3), we get the problem: find $(\boldsymbol{u}, p) \in \boldsymbol{V} \times Q$ such that

$$
a((\boldsymbol{u}, p),(\boldsymbol{v}, q))=l(q) \quad \forall(\boldsymbol{v}, q) \in \boldsymbol{V} \times Q
$$

where

$$
\begin{aligned}
a((\boldsymbol{u}, p),(\boldsymbol{v}, q)) & =\left(\boldsymbol{u}_{t}, \boldsymbol{v}_{t}\right)_{\Gamma}+\left(u_{n}, v_{n}\right)_{\Gamma}+\left(\nabla_{\Gamma} p, \boldsymbol{v}_{t}\right)_{\Gamma}-\left(\boldsymbol{u}_{t}, \nabla_{\Gamma} q\right)_{\Gamma} \\
& =(\boldsymbol{u}, \boldsymbol{v})_{\Gamma}-\left(p, \operatorname{div}_{\Gamma} \boldsymbol{v}_{t}\right)_{\Gamma}+\left(\operatorname{div}_{\Gamma} \boldsymbol{u}_{t}, q\right)_{\Gamma} \\
& =(\boldsymbol{u}, \boldsymbol{v})_{\Gamma}-\left(p, \operatorname{div}_{\Gamma} \boldsymbol{v}\right)_{\Gamma}+\left(p, H v_{n}\right)_{\Gamma}+\left(\operatorname{div}_{\Gamma} \boldsymbol{u}, q\right)_{\Gamma}-\left(u_{n} H, q\right)_{\Gamma}
\end{aligned}
$$

and $\boldsymbol{V}=\left\{\boldsymbol{v}: \Gamma \rightarrow \mathbb{R}^{3}: \boldsymbol{v} \in\left[L^{2}(\Gamma)\right]^{3}\right\}$ is the space of general $L^{2}(\Gamma)$ vector fields.

We note that $\left(\nabla_{\Gamma} p, \boldsymbol{v}\right)_{\Gamma}=\left(\nabla_{\Gamma} p, \boldsymbol{v}_{t}\right)_{\Gamma}$, since $\nabla_{\Gamma} p$ is tangential, and therefore we get a weak enforcement of the tangential condition $u_{n}=\boldsymbol{u} \cdot \boldsymbol{n}=0$ by setting $q=0$ and $\boldsymbol{v}=v_{n} \boldsymbol{n}$. Testing instead with a tangential vector field $\boldsymbol{v}_{t} \in \boldsymbol{V}_{t} \subset \boldsymbol{V}$ and $q \in Q$ we recover the Darcy problem (2.9).

Remark 2.1 We note that we have the identity

$$
\begin{aligned}
a((\boldsymbol{u}, p),(\boldsymbol{v}, q)) & =(\boldsymbol{u}, \boldsymbol{v})_{\Gamma}+\left(\nabla_{\Gamma} p, \boldsymbol{v}_{t}\right)_{\Gamma}-\left(\boldsymbol{u}_{t}, \nabla_{\Gamma} q\right)_{\Gamma} \\
& =(\boldsymbol{u}, \boldsymbol{v})_{\Gamma}-\left(p, \operatorname{div}_{\Gamma} \boldsymbol{v}_{t}\right)_{\Gamma}+\left(\operatorname{div}_{\Gamma} \boldsymbol{u}_{t}, q\right)_{\Gamma} \\
& =(\boldsymbol{u}, \boldsymbol{v})_{\Gamma}-\left(p, \operatorname{div}_{\Gamma} \boldsymbol{v}\right)_{\Gamma}+\left(p, H v_{n}\right)_{\Gamma}+\left(\operatorname{div}_{\Gamma} \boldsymbol{u}, q\right)_{\Gamma}-\left(u_{n} H, q\right)_{\Gamma}
\end{aligned}
$$

where we used the identity (2.4) for the surface divergence of a general vector field in the last step. We note that the third form (2.20) involves quantities that are directly computable while the second form (2.19) involves the surface divergence of the tangent component $\operatorname{div}_{\Gamma} \boldsymbol{v}_{t}$, which is more complicated to compute. When constructing a numerical method based on the divergence form (2.20) the term $\left(v_{n}, H q\right)_{\Gamma}$ either has to be included, which involves computation of $H$, or alternatively a stronger penalty on the normal component $v_{n}$ must be added in order to control the inconsistency resulting from neglecting the term. Neither alternative is attractive.

\subsection{Masud-Hughes Stabilized Weak Formulation}

The Masud-Hughes weak formulation, originally proposed in [12] for planar domains, for the surface Darcy problem with a general vector field velocity takes the form: find $(\boldsymbol{u}, p) \in$ $\boldsymbol{V} \times Q$ such that

$$
A((\boldsymbol{u}, p),(\boldsymbol{v}, q))=L((\boldsymbol{v}, q)) \quad \forall(\boldsymbol{v}, q) \in \boldsymbol{V} \times Q
$$


where

$$
\begin{aligned}
& A((\boldsymbol{u}, p),(\boldsymbol{v}, q))=(\boldsymbol{u}, \boldsymbol{v})_{\Gamma} \\
&+\left(\nabla_{\Gamma} p, \boldsymbol{v}\right)_{\Gamma}-\left(\boldsymbol{u}, \nabla_{\Gamma} q\right)_{\Gamma} \\
&+\frac{1}{2}\left(\boldsymbol{u}+\nabla_{\Gamma} p,-\boldsymbol{v}+\nabla_{\Gamma} q\right)_{\Gamma} \\
& L((\boldsymbol{v}, q))=\left(f^{e}, q\right)_{\Gamma}+\left(\boldsymbol{g}^{e}, \boldsymbol{v}\right)_{\Gamma}+\frac{1}{2}\left(\boldsymbol{g}^{e},-\boldsymbol{v}+\nabla_{\Gamma} q\right)_{\Gamma}
\end{aligned}
$$

Expanding the forms we obtain

$$
\begin{aligned}
A((\boldsymbol{u}, p),(\boldsymbol{v}, q)) & =\frac{1}{2}(\boldsymbol{u}, \boldsymbol{v})_{\Gamma}+\frac{1}{2}\left(\nabla_{\Gamma} p, \nabla_{\Gamma} q\right)_{\Gamma}+\frac{1}{2}\left(\nabla_{\Gamma} p, \boldsymbol{v}\right)_{\Gamma}-\frac{1}{2}\left(\boldsymbol{u}, \nabla_{\Gamma} q\right)_{\Gamma} \\
L((\boldsymbol{v}, q)) & =\left(f^{e}, q\right)_{\Gamma}+\frac{1}{2}\left(\boldsymbol{g}^{e}, \boldsymbol{v}+\nabla_{\Gamma} q\right)_{\Gamma}
\end{aligned}
$$

and thus $A$ has consists of a symmetric and a skew symmetric part.

\section{The Finite Element Method}

\subsection{Triangulation of the Surface}

Parametric Triangulated Surfaces. $\quad$ Let $\widehat{K} \subset \mathbb{R}^{2}$ be a reference triangle and let $P_{k_{g}}(\widehat{K})$ be the space of polynomials of order less or equal to $k_{g}$ defined on $\widehat{K}$. Let $\Gamma_{h, k_{g}}$ be a triangulated surface with quasi uniform triangulation $\mathcal{K}_{h, k_{g}}$ and mesh parameter $h \in\left(0, h_{0}\right]$ such that each triangle $K=F_{K, k_{g}}(\widehat{K})$ where $F_{K, k_{g}} \in\left[P_{k_{g}}(\widehat{K})\right]^{3}$. Let $\boldsymbol{n}_{h}$ be the elementwise defined normal to $\Gamma_{h}$. We let $\mathcal{E}_{h, k_{g}}$ denote the set of edges in the triangulation. For simplicity we use the notation $\mathcal{K}_{h}=\mathcal{K}_{h, k_{g}}, \mathcal{E}_{h}=\mathcal{E}_{h, k_{g}}$, and $\Gamma_{h}=\Gamma_{h, k_{g}}$ when appropriate.

Geometry Approximation Property. We assume that the family $\left\{\Gamma_{h, k_{g}}, h \in\left(0, h_{0}\right]\right\}$ approximates $\Gamma$ in the following way

- $\Gamma_{h, k_{g}} \subset U_{\delta_{0}}(\Gamma)$ and $\boldsymbol{p}: \Gamma_{h, k_{g}} \rightarrow \Gamma$ is a bijection.

- The following estimates hold

$$
\|\boldsymbol{p}\|_{L^{\infty}\left(\Gamma_{h, k_{g}}\right)} \lesssim h^{k_{g}+1}, \quad\left\|\boldsymbol{n} \circ \boldsymbol{p}-\boldsymbol{n}_{h}\right\|_{L^{\infty}\left(\Gamma_{h, k_{g}}\right)} \lesssim h^{k_{g}}
$$

These properties are valid, e.g., if $F_{K, k_{g}}$ is constructed using Lagrange interpolation of the surface. 


\subsection{Parametric Finite Element Spaces}

Let

$$
V_{h, k, k_{g}}=\left\{\boldsymbol{v}:\left.\boldsymbol{v}\right|_{K} \circ F_{K, k_{g}} \in P_{k}(\hat{K}), \forall K \in \mathcal{K}_{h, k_{g}} ; \boldsymbol{v} \in\left[C^{0}\left(\Gamma_{h}\right)\right]^{3}\right\}
$$

be the space of parametric continuous piecewise polynomials of order $k$ mapped with a mapping of order $k_{g}$. We let

$$
\boldsymbol{V}_{h}=\left[V_{h, k_{u}, k_{g}}\right]^{3}, \quad Q_{h}=\left\{q \in V_{h, k_{p}, k_{g}}: \int_{\Gamma_{h, k_{g}}} q=0\right\}
$$

be the finite element spaces for velocity and pressure, consisting of continuous piecewise polynomials of order $k_{u}$ and $k_{p}$, respectively, with parametric map of order $k_{g}$ (which is the same for both spaces).

\subsection{Interpolation}

Let

$$
\pi_{h, 1}: L^{2}\left(\mathcal{K}_{h, 1}\right) \ni v \mapsto \pi_{h, 1} v \in V_{h, k, 1}
$$

be a Scott-Zhang type interpolant. Then, for each element $K \in \mathcal{K}_{h, 1}$ we have the following elementwise estimate

$$
\left\|v^{e}-\pi_{h, 1} v^{e}\right\|_{H^{m}(K)} \lesssim h^{s-m}\left\|v^{e}\right\|_{H^{s}(N(K))} \lesssim\|v\|_{H^{s}\left(N^{l}(K)\right)}, \quad m \leq s \leq k+1, \quad m=0,1
$$

where $N(K)$ is the union of the neighboring elements to element $K$ and $N^{l}(K)=(N(K))^{l}$. In (3.5) the first inequality follows from interpolation theory, see [2], and the second from the chain rule in combination with $L^{\infty}$ boundedness of derivatives of the closest point map $\boldsymbol{p}$ in the tubular neighborhood $U_{\delta_{0}}(\Gamma)$ which follows from smoothness of $\Gamma$.

Next we define the interpolant $\pi_{h, k_{g}}: L^{2}\left(\mathcal{K}_{h}\right) \rightarrow V_{h, 1, k_{g}}$ as follows

$$
\left.\pi_{h, k_{g}} v^{e}\right|_{K}=\left(\pi_{h, 1} v^{e}\right) \circ G_{K, k_{g}, 1}
$$

where $G_{K, k_{g}, 1}=F_{K, 1} \circ F_{K, k_{g}}^{-1}: K_{k_{g}} \rightarrow K_{1}$ is a bijection from the curved triangle $K_{k_{g}}$ to the corresponding flat triangle $K_{1}$. Using uniform $L^{\infty}$ bounds on $G_{K, k_{g}, 1}$ and its first order derivative we have the estimates

$$
\begin{aligned}
\left\|v^{e}-\pi_{h, k_{g}} v^{e}\right\|_{H^{m}\left(K_{k_{g}}\right)} \lesssim & \left\|v^{e}-\pi_{h, 1} v^{e}\right\|_{H^{m}\left(K_{1}\right)} \\
& \lesssim h^{s-m}\left\|v^{e}\right\|_{H^{s}\left(N\left(K_{1}\right)\right)} \lesssim h^{s-m}\|v\|_{H^{s}\left(N^{l}\left(K_{1}\right)\right)}
\end{aligned}
$$

and thus we conclude that we have the estimate

$$
\left\|v^{e}-\pi_{h, k_{g}} v^{e}\right\|_{H^{m}\left(K_{k g}\right)} \lesssim h^{s-m}\|v\|_{H^{s}\left(N^{l}\left(K_{1}\right)\right)}, \quad m \leq s \leq k+1, \quad m=0,1
$$

for all $K \in \mathcal{K}_{h, k_{g}}$. We also have the stability estimate

$$
\left\|\pi_{h, k_{g}} v^{e}\right\|_{H^{m}\left(K_{k_{g}}\right)} \lesssim\|v\|_{H^{m}\left(N^{l}\left(K_{1}\right)\right)}, \quad m=0,1
$$


When appropriate we simplify the notation and write $\pi_{h}=\pi_{h, k_{g}}$.

Finally, we have the super-approximation result

$$
\left\|\left(I-\pi_{h}\right)\left(\chi^{e} v\right)\right\|_{\Gamma_{h}} \lesssim h\|\chi\|_{W_{\infty}^{k_{g}+1}(\Gamma)}\|v\|_{\Gamma_{h}}
$$

for $\chi \in W_{\infty}^{k_{g}+1}(\Gamma)$ and $v \in V_{h, k_{g}}$.

\subsection{Masud-Hughes Stabilized Finite Element Method}

The finite element method based on the Masud-Hughes weak formulation (2.21) for the surface Darcy problem takes the form: find $\left(\boldsymbol{u}_{h}, p_{h}\right) \in \boldsymbol{V}_{h} \times Q_{h}$ such that

$$
A_{h}\left(\left(\boldsymbol{u}_{h}, p_{h}\right),(\boldsymbol{v}, q)\right)=L_{h}((\boldsymbol{v}, q)) \quad \forall(\boldsymbol{v}, q) \in \boldsymbol{V}_{h} \times Q_{h}
$$

where

$$
\begin{aligned}
A_{h}((\boldsymbol{u}, p),(\boldsymbol{v}, q))= & (\boldsymbol{u}, \boldsymbol{v})_{\Gamma_{h}} \\
& +\left(\nabla_{\Gamma_{h}} p, \boldsymbol{v}\right)_{\Gamma_{h}}-\left(\boldsymbol{u}, \nabla_{\Gamma_{h}} q\right)_{\Gamma_{h}} \\
& +\frac{1}{2}\left(\boldsymbol{u}+\nabla_{\Gamma_{h}} p,-\boldsymbol{v}+\nabla_{\Gamma_{h}} q\right)_{\Gamma_{h}} \\
= & \frac{1}{2}(\boldsymbol{u}, \boldsymbol{v})_{\Gamma_{h}}+\frac{1}{2}\left(\nabla_{\Gamma_{h}} p, \nabla_{\Gamma_{h}} q\right)_{\Gamma_{h}}+\frac{1}{2}\left(\nabla_{\Gamma_{h}} p, \boldsymbol{v}\right)_{\Gamma_{h}}-\frac{1}{2}\left(\boldsymbol{u}, \nabla_{\Gamma_{h}} q\right)_{\Gamma_{h}} \\
L_{h}((\boldsymbol{v}, q))= & \left(f^{e}, q\right)_{\Gamma_{h}}+\left(\boldsymbol{g}^{e}, \boldsymbol{v}\right)_{\Gamma_{h}}+\frac{1}{2}\left(\boldsymbol{g}^{e},-\boldsymbol{v}+\nabla_{\Gamma_{h}} q\right)_{\Gamma_{h}} \\
= & \left(f^{e}, q\right)_{\Gamma_{h}}+\frac{1}{2}\left(\boldsymbol{g}^{e}, \boldsymbol{v}+\nabla_{\Gamma_{h}} q\right)_{\Gamma_{h}}
\end{aligned}
$$

Remark 3.1 We could add the term $c_{N}\left(\boldsymbol{n}_{h} \cdot \boldsymbol{u}, \boldsymbol{n}_{h} \cdot \boldsymbol{v}\right)_{\Gamma_{h}}$, where $c_{N} \geq 0$ is a parameter, to enforce the normal constraint more strongly. We will, however, see that we can take $c_{N}=0$, and no significant advantages of taking $c_{N}>0$ has been observed in our numerical experiments.

\section{Preliminary Results}

\subsection{Extension and Lifting of Functions}

In this section we summarize basic results concerning extension and liftings of functions. We refer to [3] and [4] for further details.

Extension. Recalling the definition $v^{e}=v \circ \boldsymbol{p}$ of the extension and using the chain rule we obtain the identity

$$
\nabla_{\Gamma_{h}} v^{e}=\boldsymbol{B}_{t}^{T} \nabla_{\Gamma} v
$$


where

$$
\boldsymbol{B}_{t}=\boldsymbol{P}_{\Gamma}(\boldsymbol{I}-\rho \boldsymbol{\kappa}) \boldsymbol{P}_{\Gamma_{h}}: T_{\boldsymbol{x}}(K) \rightarrow T_{\boldsymbol{p}(\boldsymbol{x})}(\Gamma)
$$

and we recall that $\boldsymbol{\kappa}=\nabla \otimes \nabla \rho$ which may be expressed in the form

$$
\boldsymbol{\kappa}(\boldsymbol{x})=\sum_{i=1}^{2} \frac{\kappa_{i}^{e}}{1+\rho(\boldsymbol{x}) \kappa_{i}^{e}} \boldsymbol{a}_{i}^{e} \otimes \boldsymbol{a}_{i}^{e}
$$

where $\kappa_{i}$ are the principal curvatures with corresponding orthonormal principal curvature vectors $\boldsymbol{a}_{i}$, see [8] Lemma 14.7. We note that there is $\delta>0$ such that the uniform bound

$$
\|\boldsymbol{\kappa}\|_{L^{\infty}\left(U_{\delta}(\Gamma)\right)} \lesssim 1
$$

holds. Furthermore, we have the inverse mapping

$$
\boldsymbol{B}_{t}^{-1}=\boldsymbol{P}_{\Gamma_{h}}(\boldsymbol{I}-\rho \boldsymbol{\kappa})^{-1} \boldsymbol{P}_{\Gamma}: T_{\boldsymbol{p}(\boldsymbol{x})}(\Gamma) \rightarrow T_{\boldsymbol{x}}(K)
$$

We extend $\boldsymbol{B}_{t}$ to $T_{\boldsymbol{x}}(K) \oplus N_{\boldsymbol{x}}(K)$, where $N_{\boldsymbol{x}}(K)$ is the vector space of vector fields that are normal to $K$ at $\boldsymbol{x} \in K$, by defining

$$
\boldsymbol{B}=\left(\boldsymbol{P}_{\Gamma} \boldsymbol{B}_{t} \boldsymbol{P}_{\Gamma_{h}}+\boldsymbol{n} \otimes \boldsymbol{n}_{h}\right)
$$

with inverse

$$
\boldsymbol{B}^{-1}=\left(\boldsymbol{P}_{\Gamma_{h}} \boldsymbol{B}_{t}^{-1} \boldsymbol{P}_{\Gamma}+\boldsymbol{n}_{h} \otimes \boldsymbol{n}\right)
$$

We note that $\boldsymbol{B}$ and $\boldsymbol{B}^{-1}$ preserves the tangent and normal spaces as follows

$$
\boldsymbol{B} T_{\boldsymbol{x}}(K)=T_{\boldsymbol{p}(\boldsymbol{x})}(\Gamma), \quad \boldsymbol{B} N_{\boldsymbol{x}}(K)=N_{\boldsymbol{p}(\boldsymbol{x})}(\Gamma)
$$

and

$$
\boldsymbol{B}^{-1} T_{\boldsymbol{p}(\boldsymbol{x})}(\Gamma)=T_{\boldsymbol{x}}(K), \quad \boldsymbol{B}^{-1} N_{\boldsymbol{p}(\boldsymbol{x})}(\Gamma)=N_{\boldsymbol{x}}(K)
$$

For clarity, we will employ the notation $\boldsymbol{B}(\boldsymbol{p}(\boldsymbol{x}))=\boldsymbol{B}(\boldsymbol{x})$ for each $\boldsymbol{x} \in K, K \in \mathcal{K}_{h}$, so that we do not have to indicate lift or extensions of the operator $\boldsymbol{B}$.

Lifting. The lifting $w^{l}$ of a function $w$ defined on $\Gamma_{h}$ to $\Gamma$ is defined as the push forward

$$
\left(w^{l}\right)^{e}=w^{l} \circ \boldsymbol{p}=w \quad \text { on } \Gamma_{h}
$$

and we have the identity

$$
\nabla_{\Gamma} w^{l}=\boldsymbol{B}^{-T}\left(\nabla_{\Gamma_{h}} w\right)^{l}
$$




\subsection{Estimates Related to $B$}

Using the uniform bound (4.4) it follows that

$$
\|\boldsymbol{B}\|_{L^{\infty}\left(\Gamma_{h}\right)} \lesssim 1, \quad\left\|\boldsymbol{B}^{-1}\right\|_{L^{\infty}(\Gamma)} \lesssim 1
$$

Furthermore, we have the estimates

$$
\|\boldsymbol{B}-\boldsymbol{I}\|_{L^{\infty}\left(\Gamma_{h}\right)} \lesssim h^{k_{g}} \quad\left\|\boldsymbol{I}-\boldsymbol{B}^{T} \boldsymbol{B}\right\|_{L^{\infty}\left(\Gamma_{h}\right)} \lesssim h^{k_{g}+1}
$$

To prove the first estimate in 4.13 we note, using the definition 4.2 of $\boldsymbol{B}$ and the bound (3.1) on $\rho$ that

$$
\boldsymbol{B}=\boldsymbol{P}_{\Gamma} \boldsymbol{P}_{\Gamma_{h}}+\boldsymbol{n} \otimes \boldsymbol{n}_{h}+O\left(h^{k_{g}+1}\right)
$$

Next writing $\boldsymbol{I}=\boldsymbol{P}_{\Gamma}+\boldsymbol{n} \otimes \boldsymbol{n}=\boldsymbol{P}_{\Gamma}^{2}+\boldsymbol{n} \otimes \boldsymbol{n}$, where we used that $\boldsymbol{P}_{\Gamma}$ is a projection, we obtain

$$
\boldsymbol{B}-\boldsymbol{I}=\boldsymbol{P}_{\Gamma}\left(\boldsymbol{P}_{\Gamma_{h}}-\boldsymbol{P}_{\Gamma}\right)+\boldsymbol{n} \otimes\left(\boldsymbol{n}_{h}-\boldsymbol{n}\right)+O\left(h^{k_{g}+1}\right)
$$

and thus the estimate follows using (3.1) since $\left\|\boldsymbol{P}_{\Gamma}-\boldsymbol{P}_{\Gamma_{h}}\right\|_{L^{\infty}\left(\Gamma_{h}\right)} \lesssim\left\|\boldsymbol{n}-\boldsymbol{n}_{h}\right\|_{L^{\infty}\left(\Gamma_{h}\right)} \lesssim h^{k_{g}}$.

For the second estimate in (4.13) we use the identities $\boldsymbol{I}=\boldsymbol{P}_{\Gamma_{h}}+\boldsymbol{n}_{h} \otimes \boldsymbol{n}_{h}$ and (4.14) to conclude that

$$
\boldsymbol{B}^{T} \boldsymbol{B}-\boldsymbol{I}=\boldsymbol{P}_{\Gamma_{h}} \boldsymbol{P}_{\Gamma} \boldsymbol{P}_{\Gamma_{h}}+\boldsymbol{n}_{h} \otimes \boldsymbol{n}_{h}-\boldsymbol{I}+O\left(h^{k_{g}+1}\right)=\boldsymbol{P}_{\Gamma_{h}} \boldsymbol{P}_{\Gamma} \boldsymbol{P}_{\Gamma_{h}}-\boldsymbol{P}_{\Gamma_{h}}+O\left(h^{k_{g}+1}\right)
$$

Now

$$
\boldsymbol{P}_{\Gamma_{h}} \boldsymbol{P}_{\Gamma} \boldsymbol{P}_{\Gamma_{h}}-\boldsymbol{P}_{\Gamma_{h}}=\left(\boldsymbol{P}_{\Gamma_{h}} \boldsymbol{n}\right) \otimes\left(\boldsymbol{P}_{\Gamma_{h}} \boldsymbol{n}\right)=\left(\boldsymbol{P}_{\Gamma_{h}}\left(\boldsymbol{n}-\boldsymbol{n}_{h}\right)\right) \otimes\left(\boldsymbol{P}_{\Gamma_{h}}\left(\boldsymbol{n}-\boldsymbol{n}_{h}\right)\right) \sim O\left(h^{2 k_{g}}\right)
$$

where we used the bound for the error in the discrete normal (3.1). Thus the second bound in 4.13 follows.

Further, the surface measure $d \Gamma=|\boldsymbol{B}| d \Gamma_{h}$, where $|\boldsymbol{B}|=|\operatorname{det}(\boldsymbol{B})|$ is the absolute value of the determinant of $\boldsymbol{B}$ and we have the following estimates

$$
\left\|1-\left|\boldsymbol{B}\left\|_{L^{\infty}\left(\Gamma_{h}\right)} \lesssim h^{k_{g}+1}, \quad\right\|\right| \boldsymbol{B}\right\|_{L^{\infty}\left(\Gamma_{h}\right)} \lesssim 1, \quad\left\||\boldsymbol{B}|^{-1}\right\|_{L^{\infty}\left(\Gamma_{h}\right)} \lesssim 1
$$

\subsection{Norm Equivalences}

In view of the bounds in Section 4.2 and the identities 4.1) and 4.11) we obtain the following equivalences

$$
\left\|v^{l}\right\|_{L^{2}(\Gamma)} \sim\|v\|_{L^{2}\left(\Gamma_{h}\right)}, \quad\|v\|_{L^{2}(\Gamma)} \sim\left\|v^{e}\right\|_{L^{2}\left(\Gamma_{h}\right)}
$$

and

$$
\left\|\nabla_{\Gamma} v^{l}\right\|_{L^{2}(\Gamma)} \sim\left\|\nabla_{\Gamma_{h}} v\right\|_{L^{2}\left(\Gamma_{h}\right)}, \quad\left\|\nabla_{\Gamma} v\right\|_{L^{2}(\Gamma)} \sim\left\|\nabla_{\Gamma_{h}} v^{e}\right\|_{L^{2}\left(\Gamma_{h}\right)}
$$




\subsection{Poincaré Inequality}

We have the following Poincaré inequality

$$
\|v\|_{\Gamma_{h}} \lesssim\left\|\nabla_{\Gamma_{h}} v\right\|_{\Gamma_{h}} \quad \forall v \in Q_{h}
$$

To prove 4.21 we let

$$
\lambda_{S}(v)=|S|^{-1} \int_{S} v, \quad S \in\left\{\Gamma, \Gamma_{h}\right\}
$$

be the average over $S$. Using the fact that $\alpha=\lambda_{\Gamma_{h}}(v)$ is the constant that minimizes

$\|v-\alpha\|_{\Gamma_{h}}$, norm equivalence (4.19) to pass from $\Gamma_{h}$ to $\Gamma$, the standard Poincaré estimate on $\Gamma$, and at last norm equivalence 4.20 to pass back to $\Gamma_{h}$, we obtain

$$
\left\|v-\lambda_{\Gamma_{h}}(v)\right\|_{\Gamma_{h}} \leq\left\|v-\lambda_{\Gamma}\left(v^{l}\right)\right\|_{\Gamma_{h}} \lesssim\left\|v^{l}-\lambda_{\Gamma}\left(v^{l}\right)\right\|_{\Gamma} \lesssim\left\|\nabla_{\Gamma} v^{l}\right\|_{\Gamma} \lesssim\left\|\nabla_{\Gamma_{h}} v\right\|_{\Gamma_{h}}
$$

which proves 4.21.

\section{$5 \quad$ Error Estimates}

\section{$5.1 \quad$ Norms}

Let

$$
\|(\boldsymbol{v}, q)\|^{2}=\|\boldsymbol{v}\|_{\Gamma}^{2}+\left\|\nabla_{\Gamma} q\right\|_{\Gamma}^{2}, \quad\|\|(\boldsymbol{v}, q)\|\|_{h}^{2}=\|\boldsymbol{v}\|_{\Gamma_{h}}^{2}+\left\|\nabla_{\Gamma_{h}} q\right\|_{\Gamma_{h}}^{2}
$$

Using 4.19 and 4.20 we have the following equivalences

$$
\left\|\left|\left(\boldsymbol{v}^{l}, q^{l}\right)\right|\right\| \sim\left\|( \boldsymbol { v } , q ) \left|\left\|_{h}, \quad\right\|\left\|(\boldsymbol{v}, q)|\|\sim\||\left(\boldsymbol{v}^{e}, q^{e}\right)\right\|_{h}\right.\right.
$$

\subsection{Coercivity and Continuity}

Lemma 5.1 The following statements hold:

- The form $A_{h}$ is coercive and continuous

$$
\begin{array}{cc}
\|\mid(\boldsymbol{v}, q)\|_{h}^{2} \lesssim A_{h}((\boldsymbol{v}, q),(\boldsymbol{v}, q)) & \forall(\boldsymbol{v}, q) \in \boldsymbol{V}_{h} \times Q_{h} \\
A_{h}((\boldsymbol{v}, q),(\boldsymbol{w}, r)) \lesssim\|\mid(\boldsymbol{v}, q)\|\left\|_{h}\right\|\|(\boldsymbol{w}, r)\|_{h} & \forall(\boldsymbol{v}, q),(\boldsymbol{w}, r) \in \boldsymbol{V}_{h} \times Q_{h}
\end{array}
$$

for all $h \in\left(0, h_{0}\right]$.

- The form $L_{h}$ is continuous

$$
L_{h}((\boldsymbol{v}, q)) \lesssim\left(\|f\|_{\Gamma}+\|\boldsymbol{g}\|_{\Gamma}\right)\|\|(\boldsymbol{v}, q) \|_{h} \quad \forall(\boldsymbol{v}, q) \in \boldsymbol{V}_{h} \times Q_{h}
$$

for all $h \in\left(0, h_{0}\right]$.

- There exists a unique solution to (3.12). 
Proof. Coercivity of $A_{h}$ follows directly from the definition (3.13) of the stabilized bilinear form. Continuity of $A_{h}$ follows from the Cauchy-Schwarz inequality. Continuity of $L_{h}$ follows directly from the Cauchy-Schwarz inequality and the Poincaré inequality. Existence and uniqueness of a solution to (3.12) follows from the Lax-Milgram lemma.

Remark 5.1 Clearly the analogous results holds for the continuous forms $A$ and $L$ on $\boldsymbol{V} \times Q$, defined in (2.22) and (2.23), and the variational problem 2.21).

\subsection{Discrete Stability Estimate}

Lemma 5.2 The solution $\left(\boldsymbol{u}_{h}, p_{h}\right)$ to (3.12), satisfies the stability estimate

$$
\left\|\left(\boldsymbol{u}_{h}, p_{h}\right) \mid\right\|_{h}+h^{-1}\left\|\boldsymbol{n} \cdot \boldsymbol{u}_{h}\right\|_{\Gamma_{h}} \lesssim\|f\|_{\Gamma}+\|\boldsymbol{g}\|_{\Gamma}
$$

Proof. First Test Function. With $(\boldsymbol{v}, q)=\left(\boldsymbol{u}_{h}, p_{h}\right)$ in (3.12) we obtain

$$
\left\|||\left(\boldsymbol{u}_{h}, p_{h}\right)\right\|\left\|_{h}^{2} \lesssim A_{h}\left(\left(\boldsymbol{u}_{h}, p_{h}\right),\left(\boldsymbol{u}_{h}, p_{h}\right)\right)=L_{h}\left(\left(\boldsymbol{u}_{h}, p_{h}\right)\right) \lesssim\left(\|f\|_{\Gamma}+\|\boldsymbol{g}\|_{\Gamma}\right)\right\|\left(\boldsymbol{u}_{h}, p_{h}\right)\|\|_{h}
$$

where we used (5.3) and (5.4) together with the continuity

$$
L_{h}((\boldsymbol{v}, q)) \lesssim\left(\|f\|_{\Gamma}+\|\boldsymbol{g}\|_{\Gamma}\right)\|\|(\boldsymbol{v}, q) \|_{h}, \forall(\boldsymbol{v}, q) \in \boldsymbol{V}_{h} \times Q_{h}
$$

of $L_{h}$, which follows from Cauchy-Schwarz and the Poincaré inequality (4.21). Thus we conclude that

$$
\left\|\left(\boldsymbol{u}_{h}, p_{h}\right) \mid\right\|_{h} \lesssim\|f\|_{\Gamma}+\|\boldsymbol{g}\|_{\Gamma}
$$

Second Test Function. Setting $q=0$ in $(3.12)$ we note that the following equation holds

$$
\left(\boldsymbol{u}_{h}, \boldsymbol{v}\right)_{\Gamma_{h}}+\left(\nabla_{\Gamma_{h}} p_{h}, \boldsymbol{v}\right)_{\Gamma_{h}}=\left(\boldsymbol{g}^{e}, \boldsymbol{v}\right)_{\Gamma_{h}}, \quad \forall \boldsymbol{v} \in \boldsymbol{V}_{h}
$$

Choosing the test function $\boldsymbol{v}=\pi_{h}\left(\boldsymbol{n} \pi_{h}\left(\boldsymbol{n} \cdot \boldsymbol{u}_{h}\right)\right)$ we get the identity

$$
\underbrace{\left(\boldsymbol{u}_{h}, \pi_{h}\left(\boldsymbol{n} \pi_{h}\left(\boldsymbol{n} \cdot \boldsymbol{u}_{h}\right)\right)\right)_{\Gamma_{\boldsymbol{h}}}}_{I}+\underbrace{\left(\nabla_{\Gamma_{h}} p_{h}, \pi_{h}\left(\boldsymbol{n} \pi_{h}\left(\boldsymbol{n} \cdot \boldsymbol{u}_{h}\right)\right)\right)_{\Gamma_{h}}}_{I I}=\underbrace{\left(\boldsymbol{g}^{e}, \pi_{h}\left(\boldsymbol{n} \pi_{h}\left(\boldsymbol{n} \cdot \boldsymbol{u}_{h}\right)\right)\right)_{\Gamma_{h}}}_{I I I}
$$

Term $\boldsymbol{I}$. We have

$$
\begin{aligned}
& \left(\boldsymbol{u}_{h}, \pi_{h}\left(\boldsymbol{n} \pi_{h}\left(\boldsymbol{n} \cdot \boldsymbol{u}_{h}\right)\right)\right)_{\Gamma_{h}} \\
& \quad=\left(\boldsymbol{n} \cdot \boldsymbol{u}_{h}, \boldsymbol{n} \cdot \boldsymbol{u}_{h}\right)_{\Gamma_{h}}+\left(\pi_{h}\left(\boldsymbol{n} \pi_{h}\left(\boldsymbol{n} \cdot \boldsymbol{u}_{h}\right)\right)-\boldsymbol{n}\left(\boldsymbol{n} \cdot \boldsymbol{u}_{h}\right), \boldsymbol{n} \cdot \boldsymbol{u}_{h}\right)_{\Gamma_{h}} \\
& \quad \geq\left\|\boldsymbol{n} \cdot \boldsymbol{u}_{h}\right\|_{\Gamma_{h}}^{2}-\left\|\pi_{h}\left(\boldsymbol{n} \pi_{h}\left(\boldsymbol{n} \cdot \boldsymbol{u}_{h}\right)\right)-\boldsymbol{n}\left(\boldsymbol{n} \cdot \boldsymbol{u}_{h}\right)\right\|_{\Gamma_{h}}\left\|\boldsymbol{n} \cdot \boldsymbol{u}_{h}\right\|_{\Gamma_{h}}^{2} \\
& \quad \geq(1-\delta)\left\|\boldsymbol{n} \cdot \boldsymbol{u}_{h}\right\|_{\Gamma_{h}}^{2}-\delta^{-1} \underbrace{\left\|\pi_{h}\left(\boldsymbol{n} \pi_{h}\left(\boldsymbol{n} \cdot \boldsymbol{u}_{h}\right)\right)-\boldsymbol{n}\left(\boldsymbol{n} \cdot \boldsymbol{u}_{h}\right)\right\|_{\Gamma_{h}}^{2}}_{\star}
\end{aligned}
$$


where we used the Cauchy-Schwarz inequality and finally $a b \leq \delta a^{2}+\delta^{-1} b^{2}, \delta>0$. Estimating the second term on the right hand side by adding and subtracting suitable terms we obtain

$$
\begin{aligned}
\star & \lesssim\left\|\left(\pi_{h}-I\right)\left(\boldsymbol{n} \pi_{h}\left(\boldsymbol{n} \cdot \boldsymbol{u}_{h}\right)\right)\right\|_{\Gamma_{h}}^{2}+\left\|\boldsymbol{n}\left(\pi_{h}-I\right)\left(\boldsymbol{n} \cdot \boldsymbol{u}_{h}\right)\right\|_{\Gamma_{h}}^{2} \\
& \lesssim h^{2}\left\|\pi_{h}\left(\boldsymbol{n} \cdot \boldsymbol{u}_{h}\right)\right\|_{\Gamma_{h}}^{2}+h^{2}\left\|\boldsymbol{u}_{h}\right\|_{\Gamma_{h}}^{2} \\
& \lesssim h^{2}\left\|\boldsymbol{u}_{h}\right\|_{\Gamma_{h}}^{2}
\end{aligned}
$$

where we used the super-approximation (3.11) and in the last step the $L^{2}$ stability 3.10 of the interpolant $\pi_{h}$. We thus arrive at

$$
I \gtrsim(1-\delta)\left\|\boldsymbol{n} \cdot \boldsymbol{u}_{h}\right\|_{\Gamma_{h}}^{2}-C h^{2}\left\|\boldsymbol{u}_{h}\right\|_{\Gamma_{h}}^{2}
$$

Term II. We have

$$
\begin{aligned}
I I & \lesssim\left\|\nabla_{\Gamma_{h}} p_{h}\right\|_{\Gamma_{h}}\left\|\boldsymbol{P}_{\Gamma_{h}} \pi_{h}\left(\boldsymbol{n} \pi_{h}\left(\boldsymbol{n} \cdot \boldsymbol{u}_{h}\right)\right)\right\|_{\Gamma_{h}} \\
& \lesssim h\left\|\nabla_{\Gamma_{h}} p_{h}\right\|_{\Gamma_{h}}\left\|\boldsymbol{n} \cdot \boldsymbol{u}_{h}\right\|_{\Gamma_{h}} \\
& \leq \delta^{-1} C h^{2}\left\|\nabla_{\Gamma_{h}} p_{h}\right\|_{\Gamma_{h}}^{2}+\delta\left\|\boldsymbol{n} \cdot \boldsymbol{u}_{h}\right\|_{\Gamma_{h}}^{2}
\end{aligned}
$$

Here we used the estimate

$$
\begin{aligned}
\| \boldsymbol{P}_{\Gamma_{h}} & \pi_{h}\left(\boldsymbol{n} \pi_{h}\left(\boldsymbol{n} \cdot \boldsymbol{u}_{h}\right)\right) \|_{\Gamma_{h}} \\
& \lesssim\left\|\boldsymbol{P}_{\Gamma_{h}}\left(\pi_{h}-I\right)\left(\boldsymbol{n} \pi_{h}\left(\boldsymbol{n} \cdot \boldsymbol{u}_{h}\right)\right)\right\|_{\Gamma_{h}}+\left\|\left(\boldsymbol{P}_{\Gamma_{h}} \boldsymbol{n}\right) \pi_{h}\left(\boldsymbol{n} \cdot \boldsymbol{u}_{h}\right)\right\|_{\Gamma_{h}} \\
& \lesssim h\left\|\pi_{h}\left(\boldsymbol{n} \cdot \boldsymbol{u}_{h}\right)\right\|_{\Gamma_{h}}+\left\|\boldsymbol{P}_{\Gamma_{h}} \boldsymbol{n}\right\|_{L^{\infty}\left(\Gamma_{h}\right)}\left\|\pi_{h}\left(\boldsymbol{n} \cdot \boldsymbol{u}_{h}\right)\right\|_{\Gamma_{h}} \\
& \lesssim h\left\|\boldsymbol{n} \cdot \boldsymbol{u}_{h}\right\|_{\Gamma_{h}}+h^{k_{g}}\left\|\boldsymbol{n} \cdot \boldsymbol{u}_{h}\right\|_{\Gamma_{h}} \\
& \lesssim h\left\|\boldsymbol{n} \cdot \boldsymbol{u}_{h}\right\|_{\Gamma_{h}}
\end{aligned}
$$

where we added and subtracted $\boldsymbol{n} \pi_{h}\left(\boldsymbol{n} \cdot \boldsymbol{u}_{h}\right)$, used the triangle inequality followed by the super-approximation 3.11 and the $L^{2}$ stability 3.10 of the interpolant, and finally used the fact that $k_{g} \geq 1$ and $h \in\left(0, h_{0}\right]$.

Term III. We have

$$
\begin{aligned}
|I I I| & =\left|\left(\boldsymbol{g}^{e}, \pi_{h}\left(\boldsymbol{n} \pi_{h}\left(\boldsymbol{n} \cdot \boldsymbol{u}_{h}\right)\right)\right)_{\Gamma_{h}}\right| \\
& =\left|\left(\boldsymbol{g}^{e},\left(\pi_{h}-I\right)\left(\boldsymbol{n} \pi_{h}\left(\boldsymbol{n} \cdot \boldsymbol{u}_{h}\right)\right)\right)_{\Gamma_{h}}\right| \\
& \lesssim\left\|\boldsymbol{g}^{e}\right\|_{\Gamma_{h}}\left\|\left(\pi_{h}-I\right)\left(\boldsymbol{n} \pi_{h}\left(\boldsymbol{n} \cdot \boldsymbol{u}_{h}\right)\right)\right\|_{\Gamma_{h}} \\
& \lesssim h\left\|\boldsymbol{g}^{e}\right\|_{\Gamma_{h}}\left\|\pi_{h}\left(\boldsymbol{n} \cdot \boldsymbol{u}_{h}\right)\right\|_{\Gamma_{h}} \\
& \lesssim h\left\|\boldsymbol{g}^{e}\right\|_{\Gamma_{h}}\left\|\boldsymbol{n} \cdot \boldsymbol{u}_{h}\right\|_{\Gamma_{h}} \\
& \leq \delta^{-1} C h^{2}\left\|\boldsymbol{g}^{e}\right\|_{\Gamma_{h}}^{2}+\delta\left\|\boldsymbol{n} \cdot \boldsymbol{u}_{h}\right\|_{\Gamma_{h}}^{2}
\end{aligned}
$$

where we used the fact that $\left(\boldsymbol{g}^{e}, \boldsymbol{n}\right)_{\Gamma}=0$ to subtract $\boldsymbol{n} \pi_{h}\left(\boldsymbol{n} \cdot \boldsymbol{u}_{h}\right)$, used the CauchySchwarz inequality, used the super-approximation (3.11), and the $L^{2}$ stability (3.10) of the interpolant. 
Conclusion. Collecting the bounds (5.18), (5.21), and (5.31), for $I, I I$, and $I I I$, respectively, we arrive at

$$
\begin{aligned}
(1-3 \delta)\left\|\boldsymbol{n} \cdot \boldsymbol{u}_{h}\right\|_{\Gamma_{h}}^{2} & \lesssim \delta^{-1} h^{2}\left\|\boldsymbol{g}^{e}\right\|_{\Gamma_{h}}+\delta^{-1} h^{2}\left\|\boldsymbol{u}_{h}\right\|_{\Gamma_{h}}^{2}+\delta^{-1} h^{2}\left\|\nabla_{\Gamma_{h}} p_{h}\right\|_{\Gamma_{h}}^{2} \\
& \lesssim \delta^{-1} h^{2}\left(\left\|\boldsymbol{g}^{e}\right\|_{\Gamma_{h}}+\|\|\left(\boldsymbol{u}_{h}, p_{h}\right)\|\|_{h}^{2}\right) \\
& \lesssim \delta^{-1} h^{2}\left(\|f\|_{\Gamma}+\|\boldsymbol{g}\|_{\Gamma}\right)
\end{aligned}
$$

where we used $(5.9)$ in the last step. Choosing $\delta$ small enough completes the proof.

\subsection{Interpolation Error Estimates}

Using the interpolation error estimate (3.9) we directly obtain the following interpolation estimates in the energy norm

$$
\begin{aligned}
\left\|\left|(\boldsymbol{v}, q)-\left(\pi_{h} \boldsymbol{v}^{e}, \pi_{h} q^{e}\right)^{l}\right|\right\| & \sim\left\|(\boldsymbol{v}, q)-\left(\pi_{h} \boldsymbol{v}^{e}, \pi_{h} q^{e}\right)\right\| \|_{h} \\
& \lesssim h^{k_{u}+1}\|\boldsymbol{v}\|_{H^{k_{u}+1}(\Gamma)}+h^{k_{p}}\|q\|_{H^{k_{p}+1}(\Gamma)}
\end{aligned}
$$

for $(\boldsymbol{v}, q) \in H^{k_{u}+1}(\Gamma) \times H^{k_{p}+1}(\Gamma)$. If $\boldsymbol{v}$ is tangential, $\boldsymbol{n} \cdot \boldsymbol{v}=0$, we also have the estimate

$$
\left\|\boldsymbol{n} \cdot \pi_{h} \boldsymbol{v}\right\|_{\Gamma_{h}}=\left\|\boldsymbol{n} \cdot\left(\pi_{h} \boldsymbol{v}-\boldsymbol{v}\right)\right\|_{\Gamma_{h}} \lesssim h\|\boldsymbol{v}\|_{H^{1}(\Gamma)}
$$

\subsection{Strang's Lemma}

Lemma 5.3 Let $(\boldsymbol{u}, p)$ be the solution to (2.9) and $\left(\boldsymbol{u}_{h}, p_{h}\right)$ the solution to (3.12), then the following estimate holds

$$
\begin{aligned}
\left\|\left|(\boldsymbol{u}, p)-\left(\boldsymbol{u}_{h}, p_{h}\right)^{l}\|\mid \lesssim\|\right.\right. & \left(\boldsymbol{u}^{e}-\pi_{h} \boldsymbol{u}^{e}, p^{e}-\pi_{h} p^{e}\right)\|\|_{h} \\
& +\frac{\left.\left.A\left(\left(\pi_{h} \boldsymbol{u}^{e}, \pi_{h} p^{e}\right)^{l},(\boldsymbol{v}, q)^{l}\right)-A_{h}\left(\pi_{h} \boldsymbol{u}^{e}, \pi_{h} p^{e}\right),(\boldsymbol{v}, q)\right)\right)}{\|(\boldsymbol{v}, q)\|_{h}} \\
& +\frac{\left.L(\boldsymbol{v}, q)^{l}\right)-L_{h}((\boldsymbol{v}, q))}{\|(\boldsymbol{v}, q)\|_{h}}
\end{aligned}
$$

for all $h \in\left(0, h_{0}\right]$.

Proof. Adding and subtracting an interpolant and using the triangle inequality we obtain

$$
\begin{aligned}
\|\|(\boldsymbol{u}, p)-\left(\boldsymbol{u}_{h}^{l}, p_{h}^{l}\right)\|\| & \sim\left\|\mid\left(\boldsymbol{u}^{e}, p^{e}\right)-\left(\boldsymbol{u}_{h}, p_{h}\right)\right\| \|_{h} \\
& \lesssim\|\|\left(\boldsymbol{u}^{e}, p^{e}\right)-\left(\pi_{h} \boldsymbol{u}^{e}, \pi_{h} p^{e}\right)\left\|_{h}+\right\|\left\|\left(\pi_{h} \boldsymbol{u}^{e}, \pi_{h} p^{e}\right)-\left(\boldsymbol{u}_{h}, p_{h}\right)\right\|_{h}
\end{aligned}
$$

To estimate the second term use $(5.3)$ to conclude that

$$
\left\|\mid\left(\pi_{h} \boldsymbol{u}^{e}, \pi_{h} p^{e}\right)-\left(\boldsymbol{u}_{h}, p_{h}\right)\right\| \|_{h} \lesssim \sup _{(\boldsymbol{v}, q) \in \boldsymbol{V}_{h} \times Q_{h}} \frac{A_{h}\left(\left(\pi_{h} \boldsymbol{u}^{e}, \pi_{h} p^{e}\right)-\left(\boldsymbol{u}_{h}, p_{h}\right),(\boldsymbol{v}, q)\right)}{\|\mid(\boldsymbol{v}, q)\| \|_{h}}
$$


Using Galerkin orthogonality $(3.12)$ to eliminate $\left(\boldsymbol{u}_{h}, p_{h}\right)$, and then adding the weak form of the exact problem, the numerator may be written in the following form

$$
\begin{aligned}
& A_{h}\left(\left(\pi_{h} \boldsymbol{u}^{e}, \pi_{h} p^{e}\right)-\left(\boldsymbol{u}_{h}, u_{h}\right),(\boldsymbol{v}, q)\right) \\
& =A_{h}\left(\left(\pi_{h} \boldsymbol{u}^{e}, \pi_{h} p^{e}\right),(\boldsymbol{v}, q)\right)-L_{h}((\boldsymbol{v}, q)) \\
& =A_{h}\left(\left(\pi_{h} \boldsymbol{u}^{e}, \pi_{h} p^{e}\right),(\boldsymbol{v}, q)\right) \underbrace{-A\left((\boldsymbol{u}, p),(\boldsymbol{v}, q)^{l}\right)+L\left((\boldsymbol{v}, q)^{l}\right)}_{=0}-L_{h}((\boldsymbol{v}, q)) \\
& =A_{h}\left(\left(\pi_{h} \boldsymbol{u}^{e}, \pi_{h} p^{e}\right),(\boldsymbol{v}, q)\right)-A\left(\left(\pi_{h} \boldsymbol{u}^{e}, \pi_{h} p^{e}\right)^{l},(\boldsymbol{v}, q)^{l}\right) \\
& \quad+L\left((\boldsymbol{v}, q)^{l}\right)-L_{h}((\boldsymbol{v}, q)) \\
& \quad+A\left(\left(\left(\pi_{h} \boldsymbol{u}^{e}\right)^{l}-u,\left(\pi_{h} p^{e}\right)^{l}-p\right),(\boldsymbol{v}, q)^{l}\right)
\end{aligned}
$$

where at last we added and subtracted an interpolant. Estimating the right hand side and using (5.41) the lemma follows immediately.

\subsection{Quadrature Error Estimates}

Lemma 5.4 The following estimates hold

$$
\begin{aligned}
\left|\left(\boldsymbol{v}^{l}, \boldsymbol{w}^{l}\right)_{\mathcal{K}_{h}^{l}}-(\boldsymbol{v}, \boldsymbol{w})_{\mathcal{K}_{h}}\right| & \lesssim h^{k_{g}+1}\|\boldsymbol{v}\|_{\mathcal{K}_{h}}\|\boldsymbol{w}\|_{\mathcal{K}_{h}} \\
\left|\left(\boldsymbol{v}^{l}, \nabla_{\Gamma} q^{l}\right)_{\mathcal{K}_{h}^{l}}-\left(\boldsymbol{v}, \nabla_{\Gamma_{h}} q\right)_{\mathcal{K}_{h}}\right| & \lesssim h^{k_{g}+1}\left(\|\boldsymbol{v}\|_{\mathcal{K}_{h}}+h^{-1}\|\boldsymbol{n} \cdot \boldsymbol{v}\|_{\mathcal{K}_{h}}\right)\left\|\nabla_{\Gamma_{h}} q\right\|_{\mathcal{K}_{h}} \\
\left|\left(\nabla_{\Gamma} q^{l}, \nabla_{\Gamma} r^{l}\right)_{\mathcal{K}_{h}^{l}}-\left(\nabla_{\Gamma_{h}} q, \nabla_{\Gamma_{h}} r\right)_{\mathcal{K}_{h}}\right| & \lesssim h^{k_{g}+1}\left\|\nabla_{\Gamma_{h}} q\right\|_{\mathcal{K}_{h}^{l}}\left\|\nabla_{\Gamma_{h}} r\right\|_{\mathcal{K}_{h}} \\
\left|L\left(\left(v^{L}, q^{l}\right)\right)-L_{h}((v, q))\right| & \lesssim h^{k_{g}+1}\left(\|f\|_{\Gamma}+\|\boldsymbol{g}\|_{\Gamma}\right) \mid\|(v, q)\| \|_{h}
\end{aligned}
$$

for all $(\boldsymbol{v}, q),(\boldsymbol{w}, r) \in \boldsymbol{V}_{h} \times Q_{h}$ and $h \in\left(0, h_{0}\right]$.

Proof. (5.45): Changing domain of integration from $\mathcal{K}_{h}^{l}$ to $\mathcal{K}_{h}$ in the first term and using the bound (4.18) for $|\boldsymbol{B}|$ we obtain

$$
\begin{aligned}
\left|\left(\boldsymbol{v}^{l}, \boldsymbol{w}^{l}\right)_{\mathcal{K}_{h}^{l}}-(\boldsymbol{v}, \boldsymbol{w})_{\mathcal{K}_{h}}\right| & =\left|((|\boldsymbol{B}|-1) \boldsymbol{v}, \boldsymbol{w})_{\mathcal{K}_{h}}\right| \\
& \lesssim\||\boldsymbol{B}|-1\|_{L^{\infty}\left(\mathcal{K}_{h}\right)}\|\boldsymbol{v}\|_{\mathcal{K}_{h}}\|\boldsymbol{w}\|_{\mathcal{K}_{h}} \\
& \lesssim h^{k_{g}+1}\|\boldsymbol{v}\|_{\mathcal{K}_{h}}\|\boldsymbol{w}\|_{\mathcal{K}_{h}}
\end{aligned}
$$

(5.46): Changing domain of integration from $\mathcal{K}_{h}^{l}$ to $\mathcal{K}_{h}$ in the first term we obtain

$$
\begin{aligned}
& \left|\left(\boldsymbol{v}^{l}, \nabla_{\Gamma} q^{l}\right)_{\mathcal{K}_{h}^{l}}-\left(\boldsymbol{v}, \nabla_{\Gamma_{h}} q\right)_{\mathcal{K}_{h}}\right| \\
& \quad=\left|\left(\boldsymbol{v}^{l}, \boldsymbol{B}^{-T}\left(\nabla_{\Gamma_{h}} q\right)^{l}\right)_{\mathcal{K}_{h}^{l}}-\left(\boldsymbol{v}, \nabla_{\Gamma_{h}} q\right)_{\mathcal{K}_{h}}\right| \\
& \quad=\mid\left(\left(\boldsymbol{P}_{\Gamma_{h}}\left(|\boldsymbol{B}| \boldsymbol{B}^{-1}-\boldsymbol{I}\right) \boldsymbol{v}, \nabla_{\Gamma_{h}} q\right)_{\mathcal{K}_{h}} \mid\right.
\end{aligned}
$$


Here we have the identity

$$
\boldsymbol{P}_{\Gamma_{h}}\left(|\boldsymbol{B}| \boldsymbol{B}^{-1}-\boldsymbol{I}\right) \boldsymbol{v}=\boldsymbol{P}_{\Gamma_{h}}(|\boldsymbol{B}|-1) \boldsymbol{B}^{-1}+\boldsymbol{P}_{\Gamma_{h}}\left(\boldsymbol{B}^{-1}-\boldsymbol{I}\right)
$$

where we note that the first term i $O\left(h^{k_{g}+1}\right)$ using $(4.18)$ and the second takes the form

$$
\begin{aligned}
\boldsymbol{P}_{\Gamma_{h}}\left(\boldsymbol{B}^{-1}-\boldsymbol{I}\right) & =\boldsymbol{P}_{\Gamma_{h}}\left(\boldsymbol{P}_{\Gamma_{h}} \boldsymbol{P}_{\Gamma}+\boldsymbol{n}_{h} \otimes \boldsymbol{n}+O\left(h^{k_{g}+1}\right)-\boldsymbol{I}\right) \\
& =\boldsymbol{P}_{\Gamma_{h}} \boldsymbol{Q}_{\Gamma}+O\left(h^{k_{g}+1}\right)
\end{aligned}
$$

Thus we conclude that

$$
\begin{aligned}
\left|\left(\boldsymbol{v}^{l}, \nabla_{\Gamma} q^{l}\right)_{\mathcal{K}_{h}^{l}}-\left(\boldsymbol{v}, \nabla_{\Gamma_{h}} q\right)_{\mathcal{K}_{h}}\right| & =\mid\left(\left(\boldsymbol{P}_{\Gamma_{h}}\left(|\boldsymbol{B}| \boldsymbol{B}^{-1}-\boldsymbol{I}\right) \boldsymbol{v}, \nabla_{\Gamma_{h}} q\right)_{\mathcal{K}_{h}} \mid\right. \\
& \lesssim h^{k_{g}+1}\|\boldsymbol{v}\|_{\mathcal{K}_{h}}\left\|\nabla_{\Gamma_{h}} q\right\|_{\mathcal{K}_{h}}+\left|\left(\left(\boldsymbol{P}_{\Gamma_{h}} \boldsymbol{n}\right)(\boldsymbol{n} \cdot \boldsymbol{v}), \nabla_{\Gamma_{h}} q\right)_{\mathcal{K}_{h}}\right| \\
& \lesssim h^{k_{g}+1}\|\boldsymbol{v}\|_{\mathcal{K}_{h}}\left\|\nabla_{\Gamma_{h}} q\right\|_{\mathcal{K}_{h}}+h^{k_{g}}\|\boldsymbol{n} \cdot \boldsymbol{v}\|_{\mathcal{K}_{h}}\left\|\nabla_{\Gamma_{h}} q\right\|_{\mathcal{K}_{h}}
\end{aligned}
$$

(5.47): Using 4.11), changing domain of integration from $\mathcal{K}_{h}^{l}$ to $\mathcal{K}_{h}$ in the first term we obtain

$$
\begin{aligned}
\left|\left(\nabla_{\Gamma} q^{l}, \nabla_{\Gamma} r^{l}\right)_{\mathcal{K}_{h}^{l}}-\left(\nabla_{\Gamma_{h}} q, \nabla_{\Gamma_{h}} r\right)_{\mathcal{K}_{h}}\right| \\
\quad=\left|\left(\boldsymbol{B}^{-T}\left(\nabla_{\Gamma_{h}} q\right)^{l}, \boldsymbol{B}^{-T}\left(\nabla_{\Gamma_{h}} r\right)^{l}\right)_{\mathcal{K}_{h}^{l}}-\left(\nabla_{\Gamma_{h}} q, \nabla_{\Gamma_{h}} r\right)_{\mathcal{K}_{h}}\right| \\
\quad=\left|\left(\left(|\boldsymbol{B}| \boldsymbol{B}^{-1} \boldsymbol{B}^{-T}-\boldsymbol{I}\right) \nabla_{\Gamma_{h}} q, \nabla_{\Gamma_{h}} r\right)_{\mathcal{K}_{h}}\right| \\
\quad \lesssim\left\|\left(|\boldsymbol{B}| \boldsymbol{B}^{-1} \boldsymbol{B}^{-T}-\boldsymbol{I}\right)\right\|_{L^{\infty}\left(\mathcal{K}_{h}\right)}\left\|\nabla_{\Gamma_{h}} q\right\|_{\mathcal{K}_{h}}\left\|\nabla_{\Gamma_{h}} r\right\|_{\mathcal{K}_{h}} \\
\quad \lesssim h^{k_{g}+1}\left\|\nabla_{\Gamma_{h}} q\right\|_{\mathcal{K}_{h}}\left\|\nabla_{\Gamma_{h}} r\right\|_{\mathcal{K}_{h}}
\end{aligned}
$$

Here we used the estimate

$$
\begin{gathered}
\left\|\left(|\boldsymbol{B}| \boldsymbol{B}^{-1} \boldsymbol{B}^{-T}-\boldsymbol{I}\right)\right\|_{L^{\infty}\left(\mathcal{K}_{h}\right)} \lesssim\|\boldsymbol{B} \mid-1\|_{L^{\infty}\left(\mathcal{K}_{h}\right)}\left\|\boldsymbol{B}^{-1}\right\|_{L^{\infty}\left(\mathcal{K}_{h}\right)}\left\|\boldsymbol{B}^{-T}\right\|_{L^{\infty}\left(\mathcal{K}_{h}\right)} \\
+\left\|\boldsymbol{B}^{-1}\right\|_{L^{\infty}\left(\mathcal{K}_{h}\right)}\left\|\boldsymbol{I}-\boldsymbol{B} \boldsymbol{B}^{T}\right\|_{L^{\infty}\left(\mathcal{K}_{h}\right)}\left\|\boldsymbol{B}^{-T}\right\|_{L^{\infty}\left(\mathcal{K}_{h}\right)} \lesssim h^{k_{g}+1}
\end{gathered}
$$

where we employed (4.12), (4.13), and (4.18).

(5.48): Changing domain of integration from $\mathcal{K}_{h}^{l}$ to $\mathcal{K}_{h}$ and using (4.18) we obtain

$$
\begin{aligned}
\left|\left(f, q^{l}\right)_{\mathcal{K}_{h}^{l}}-\left(f^{e}, q\right)_{\mathcal{K}_{h}}\right|+\left|\left(\boldsymbol{g}, \boldsymbol{v}^{l}\right)_{\mathcal{K}_{h}}-\left(\boldsymbol{g}^{e}, \boldsymbol{v}\right)_{\mathcal{K}_{h}}\right| \\
\quad=\left|\left((|\boldsymbol{B}|-1) f^{e}, q\right)_{\mathcal{K}_{h}}\right|+\left|\left((|\boldsymbol{B}|-1) \boldsymbol{g}^{e}, \boldsymbol{v}\right)_{\mathcal{K}_{h}}\right| \\
\quad \lesssim\|\| \boldsymbol{B} \mid-1 \|_{L^{\infty}\left(\Gamma_{h}\right)}\left(\left\|f^{e}\right\|_{\mathcal{K}_{h}}\|q\|_{\mathcal{K}_{h}}+\left\|\boldsymbol{g}^{e}\right\|_{\mathcal{K}_{h}}\|\boldsymbol{v}\|_{\mathcal{K}_{h}}\right) \\
\quad \lesssim\||\boldsymbol{B}|-1\|_{L^{\infty}\left(\Gamma_{h}\right)}\left(\left\|f^{e}\right\|_{\mathcal{K}_{h}}^{2}+\left\|\boldsymbol{g}^{e}\right\|_{\mathcal{K}_{h}}^{2}\right)^{1 / 2}\left(\left\|\nabla_{\Gamma_{h}} q\right\|_{\mathcal{K}_{h}}^{2}+\|\boldsymbol{v}\|_{\mathcal{K}_{h}}^{2}\right)^{1 / 2} \\
\quad \lesssim h^{k_{g}+1}\left(\|f\|_{\Gamma}+\|\boldsymbol{g}\|_{\Gamma}\right)\|\mid(\boldsymbol{v}, q)\|_{h}
\end{aligned}
$$

where we used the Poincaré inequality (4.21).

We collect our results in a convenient form for the developments below in the following corollary. 
Corollary 5.1 The following estimates hold

$$
\begin{aligned}
& \left|A\left((\boldsymbol{v}, q)^{l},(\boldsymbol{w}, r)^{l}\right)-A_{h}((\boldsymbol{v}, q),(\boldsymbol{w}, r))\right| \\
& \quad \lesssim h^{k_{g}+1}\left(\|\mid\|(\boldsymbol{v}, q)\|\|_{h}+h^{-1}\|\boldsymbol{n} \cdot \boldsymbol{v}\|_{\Gamma_{h}}^{2}\right)\left(\|\|(\boldsymbol{w}, r)\|\|_{h}+h^{-1}\|\boldsymbol{n} \cdot \boldsymbol{w}\|_{\Gamma_{h}}^{2}\right)
\end{aligned}
$$

and

$$
\left|L\left((\boldsymbol{v}, q)^{l}\right)-L_{h}((\boldsymbol{v}, q))\right| \lesssim h^{k_{g}+1}\left(\|f\|_{\Gamma}+\|\boldsymbol{g}\|_{\Gamma}\right)\|||(\boldsymbol{v}, q) \mid\|_{h}
$$

for all $(\boldsymbol{v}, q)$ and $(\boldsymbol{w}, r) \in \boldsymbol{V}_{h} \times Q_{h}$ and $h \in\left(0, h_{0}\right]$.

\subsection{Error Estimates}

Theorem 5.1 Let $(\boldsymbol{u}, p)$ be the solution to (2.9) and $\left(\boldsymbol{u}_{h}, p_{h}\right)$ the solution to (3.12) and assume that the geometry approximation property holds, then for the following estimate holds

$$
\left\|\mid\left(\boldsymbol{u}-\boldsymbol{u}_{h}^{l}, p-p_{h}^{l}\right)\right\|\left\|\lesssim h^{k_{u}+1}\right\| \boldsymbol{u}\left\|_{H^{k_{u}+1}(\Gamma)}+h^{k_{p}}\right\| p \|_{H^{k_{p}+1}(\Gamma)}+h^{k_{g}}\left(\|f\|_{\Gamma}+\|\boldsymbol{g}\|_{\Gamma}\right)
$$

for all $h \in\left(0, h_{0}\right]$.

Proof. Starting from Strang's lemma we need to estimate the three terms on the right hand side in (5.38). For the first term using the interpolation estimate gives

$$
\left\|\boldsymbol{u}-\pi_{h} \boldsymbol{u}^{e}, p-\pi_{h} p^{e}\right\| \mid \lesssim h^{k_{u}+1}\|u\|_{H^{k_{u}+1}(\Gamma)}+h^{k_{p}}\|p\|_{H^{k_{p}+1}(\Gamma)}
$$

For the second term using the quadrature estimate 5.70 we obtain

$$
\begin{aligned}
& \left.\left.\mid A\left(\left(\pi_{h} \boldsymbol{u}^{e}, \pi_{h} p^{e}\right)^{l},(\boldsymbol{v}, q)^{l}\right)-A_{h}\left(\pi_{h} \boldsymbol{u}^{e}, \pi_{h} p^{e}\right),(\boldsymbol{v}, q)\right)\right) \mid \\
& \quad \lesssim h^{k_{g}+1} \underbrace{\left(\left\|\left(\pi_{h} \boldsymbol{u}^{e}, \pi_{h} p^{e}\right)\right\|\left\|_{h}+h^{-1}\right\| \boldsymbol{n} \cdot \pi_{h} \boldsymbol{u}^{e} \|_{\Gamma_{h}}\right)}_{\lesssim\|(\| \boldsymbol{u}, p)\|+\|\boldsymbol{u}\|_{H^{1}(\Gamma)}} \underbrace{\left(\|\|(\boldsymbol{v}, q)\|\|_{h}+h^{-1}\|\boldsymbol{n} \cdot \boldsymbol{v}\|_{\Gamma_{h}}\right)}_{\lesssim h^{-1}\|(\boldsymbol{v}, q)\|_{h}} \\
& \quad \lesssim h^{k_{g}\left(\|\boldsymbol{u}\|_{H^{1}(\Gamma)}+\|p\|_{H^{1}(\Gamma)}\right)\|\|(\boldsymbol{v}, q) \|_{h}}
\end{aligned}
$$

where we used the interpolation estimate (5.37) for the first term. For the third term, applying (5.71), directly gives

$$
\left|L\left((\boldsymbol{v}, q)^{l}\right)-L_{h}(\boldsymbol{v}, q)\right| \lesssim h^{k_{g}+1}\left(\|f\|_{\Gamma}+\|\boldsymbol{g}\|_{\Gamma}\right)\|(\boldsymbol{v}, q)\|_{h}
$$

Combining the three estimates with the Strang lemma we directly obtain the desired estimate. 
Theorem 5.2 Under the same assumptions as in Theorem 5.1 the following estimate holds

$$
\left\|p-p_{h}^{l}\right\|_{\Gamma} \lesssim h^{k_{u}+2}\|\boldsymbol{u}\|_{H^{k_{u}+1}(\Gamma)}+h^{k_{p}+1}\|p\|_{H^{k_{p}+1}(\Gamma)}+h^{k_{g}+1}\left(\|f\|_{\Gamma}+\|\boldsymbol{g}\|_{\Gamma}\right)
$$

for all $h \in\left(0, h_{0}\right]$.

Proof. Recall that $\lambda_{S}(v)=|S|^{-1} \int_{S} v$ is the average of a function in $L^{2}(S), S \in\left\{\Gamma, \Gamma_{h}\right\}$, see (4.22). Then we have

$$
\left\|p-p_{h}^{l}\right\|_{\Gamma} \leq \underbrace{\left\|p-\left(p_{h}^{l}-\lambda_{\Gamma}\left(p_{h}^{l}\right)\right)\right\|_{\Gamma}}_{I}+\underbrace{\left|\lambda_{\Gamma}\left(p_{h}^{l}\right)-\lambda_{\Gamma_{h}}\left(p_{h}\right)\right|}_{I I}
$$

where we added and subtracted $\lambda_{\Gamma}\left(p_{h}^{l}\right)$ and used the fact that $\lambda_{\Gamma_{h}}\left(p_{h}\right)=0$.

Term $\boldsymbol{I}$. Let $(\phi, \chi) \in \boldsymbol{V}_{t} \times Q$ be the solution to the continuous dual problem

$$
\begin{aligned}
-\operatorname{div}_{\Gamma} \phi & =\psi & & \text { on } \Gamma \\
-\phi_{t}+\nabla_{\Gamma} \chi & =\mathbf{0} & & \text { on } \Gamma
\end{aligned}
$$

for $\psi \in L^{2}(\Gamma)$ with $\int_{\Gamma} \psi=0$. We then have the elliptic regularity bound

$$
\|\phi\|_{H^{1}(\Gamma)}+\|\chi\|_{H^{2}(\Gamma)} \lesssim\|\psi\|_{\Gamma}
$$

Furthermore, we have the weak form

$$
A((\boldsymbol{v}, q),(\boldsymbol{\phi}, \chi))=(q, \psi) \quad \forall(\boldsymbol{v}, q) \in \boldsymbol{V} \times Q
$$

and setting $(\boldsymbol{v}, q)=\left(\boldsymbol{u}-\boldsymbol{u}_{h}^{l}, p-p_{h}^{l}\right)$ we obtain the error representation formula

$$
\begin{aligned}
\left(p-p_{h}^{l}, \psi\right)= & A\left(\left(\boldsymbol{u}-\boldsymbol{u}_{h}^{l}, p-p_{h}^{l}\right),(\boldsymbol{\phi}, \chi)\right) \\
= & A\left(\left(\boldsymbol{u}-\boldsymbol{u}_{h}^{l}, p-p_{h}^{l}\right),(\boldsymbol{\phi}, \chi)-\left(\pi_{h} \boldsymbol{\phi}, \pi_{h} \chi\right)\right) \\
& +A\left(\left(\boldsymbol{u}-\boldsymbol{u}_{h}^{l}, p-p_{h}^{l}\right),\left(\pi_{h} \boldsymbol{\phi}, \pi_{h} \chi\right)\right) \\
= & A\left(\left(\boldsymbol{u}-\boldsymbol{u}_{h}^{l}, p-p_{h}^{l}\right),(\boldsymbol{\phi}, \chi)-\left(\pi_{h} \boldsymbol{\phi}, \pi_{h} \chi\right)\right) \\
& +L\left(\left(\pi_{h} \boldsymbol{\phi}, \pi_{h} \chi\right)\right)-L_{h}\left(\left(\pi_{h} \boldsymbol{\phi}, \pi_{h} \chi\right)\right) \\
& +A_{h}\left(\left(\boldsymbol{u}_{h}, p_{h}\right),\left(\pi_{h} \boldsymbol{\phi}, \pi_{h} \chi\right)\right)-A\left(\left(\boldsymbol{u}_{h}, p_{h}\right)^{l},\left(\pi_{h} \boldsymbol{\phi}, \pi_{h} \chi\right)^{l}\right) \\
= & I_{1}+I_{2}+I_{3}
\end{aligned}
$$

Term $\boldsymbol{I}_{1}$. Using the continuity of $A$, the energy error estimate (5.72), and the interpolation error estimate (5.36) we obtain

$$
\begin{aligned}
|I| & \lesssim\left|\left\|( \boldsymbol { u } - \boldsymbol { u } _ { h } ^ { l } , p - p _ { h } ^ { l } ) \left|\left\|\left|\left\|(\boldsymbol{\phi}, \chi)-\left(\pi_{h} \boldsymbol{\phi}, \pi_{h} \chi\right)^{l}\right\|\right|\right.\right.\right.\right. \\
& \lesssim\left(h^{k_{u}+1}\|\boldsymbol{u}\|_{H^{k_{u}+1}(\Gamma)}+h^{k_{p}}\|p\|_{H^{k_{p}+1}(\Gamma)}\right)\left(h\|\boldsymbol{\phi}\|_{H^{1}(\Gamma)}+h\|\chi\|_{H^{2}(\Gamma)}\right) \\
& \lesssim\left(h^{k_{u}+2}\|\boldsymbol{u}\|_{H^{k_{u}+1}(\Gamma)}+h^{k_{p}+1}\|p\|_{H^{k_{p}+1}(\Gamma)}\right)\|\psi\|_{\Gamma}
\end{aligned}
$$


Term $\boldsymbol{I}_{2}$. Using the quadrature estimate (5.71) we directly obtain

$$
|I I| \lesssim h^{k_{g}+1}\left(\|f\|_{\Gamma}+\|\boldsymbol{g}\|_{\Gamma}\right)\left\|\left(\pi_{h} \boldsymbol{\phi}, \pi_{h} \chi\right)\right\|\left\|_{h} \lesssim h^{k_{g}+1}\left(\|f\|_{\Gamma}+\|\boldsymbol{g}\|_{\Gamma}\right)\right\| \psi \|_{\Gamma}
$$

where we used stability (3.10) of the interpolant and stability (5.80) of the solution to the dual problem.

Term $\boldsymbol{I}_{3}$. Using the quadrature estimate $(5.70)$ we obtain

$$
\begin{aligned}
|I I I| & \lesssim h^{k_{g}+1}\left(\|\|\left(\boldsymbol{u}_{h}, p_{h}\right)\|\|_{h}+h^{-1}\left\|\boldsymbol{n} \cdot \boldsymbol{u}_{h}\right\|_{\Gamma_{h}}\right)\left(\|\|\left(\pi_{h} \boldsymbol{\phi}, \pi_{h} \chi\right)\|\|_{h}+h^{-1}\left\|\boldsymbol{n} \cdot \pi_{h} \boldsymbol{\phi}\right\|_{\Gamma_{h}}\right) \\
& \lesssim h^{k_{g}+1}\left(\|f\|_{\Gamma}+\|\boldsymbol{g}\|_{\Gamma}\right)\left(\|\mid(\boldsymbol{\phi}, \chi)\|\|+\| \phi \|_{H^{1}(\Gamma)}\right) \\
& \lesssim h^{k_{g}+1}\left(\|f\|_{\Gamma}+\|\boldsymbol{g}\|_{\Gamma}\right)\|\psi\|_{\Gamma}
\end{aligned}
$$

where we used, the stability $(3.10)$ of the interpolant, the estimate (5.37) for the normal component of the interpolant, and the stability $(5.80)$ of the dual problem.

Conclusion Term I. Finally, setting $\psi=p-\left(p_{h}^{l}-\lambda_{\Gamma}\left(p_{h}^{l}\right)\right) /\left\|p-\left(p_{h}^{l}-\lambda_{\Gamma}\left(p_{h}^{l}\right)\right)\right\|_{\Gamma}$, and collecting the estimates of terms $I_{1}, I_{2}$, and $I_{3}$, we obtain

$$
I \lesssim h^{k_{u}+2}\|\boldsymbol{u}\|_{H^{k_{u}+1}(\Gamma)}+h^{k_{p}+1}\|p\|_{H^{k_{p}+1}(\Gamma)}+h^{k_{g}+1}\left(\|f\|_{\Gamma}+\|\boldsymbol{g}\|_{\Gamma}\right)
$$

Term II. Changing the domain of integration from $\Gamma$ to $\Gamma_{h}$, we obtain the identity

$$
\begin{aligned}
\lambda_{\Gamma}\left(p_{h}^{l}\right)-\lambda_{\Gamma_{h}}\left(p_{h}\right) & =|\Gamma|^{-1} \int_{\Gamma} p_{h}^{l}-\left|\Gamma_{h}\right|^{-1} \int_{\Gamma_{h}} p_{h} \\
& =\int_{\Gamma_{h}}\left(|\Gamma|^{-1}|\boldsymbol{B}|-\left|\Gamma_{h}\right|^{-1}\right) p_{h}
\end{aligned}
$$

Using the estimates $|\boldsymbol{B}|=1+O\left(h^{k_{g}+1}\right)$ and $\left|\Gamma_{h}\right|=|\Gamma|+O\left(h^{k_{g}+1}\right)$, and some obvious manipulations we obtain

$$
\begin{aligned}
\left|\lambda_{\Gamma}\left(p_{h}^{l}\right)-\lambda_{\Gamma_{h}}\left(p_{h}\right)\right| \lesssim\left\|\left.||\right|^{-1}|\boldsymbol{B}|-\left|\Gamma_{h}\right|^{-1}\right\|_{L^{\infty}\left(\Gamma_{h}\right)}\left\|p_{h}\right\|_{\Gamma_{h}} \\
\\
\lesssim h^{k_{g}+1}\left\|\nabla_{\Gamma_{h}} p_{h}\right\|_{\Gamma_{h}} \lesssim h^{k_{g}+1}\left(\|f\|_{\Gamma}+\|\boldsymbol{g}\|_{\Gamma}\right)
\end{aligned}
$$

where at last we used the Poincaré estimate (4.21). Thus we conclude that

$$
I I \lesssim h^{k_{g}+1}\left(\|f\|_{\Gamma}+\|\boldsymbol{g}\|_{\Gamma}\right)
$$

Conclusion. Together the estimates (5.93) and (5.98) of Terms $I$ and $I I$ proves the desired estimate. 
Theorem 5.3 Under the same assumptions as in Theorem 5.1 the following estimate holds

$$
\left\|\boldsymbol{P}_{\Gamma}\left(\boldsymbol{u}-\boldsymbol{u}_{h}^{l}\right)\right\|_{\Gamma} \lesssim h^{k_{u}+1}\|\boldsymbol{u}\|_{H^{k_{u}+1}(\Gamma)}+h^{k_{p}}\|p\|_{H^{k_{p}+1}(\Gamma)}+h^{k_{g}+1}\left(\|f\|_{\Gamma}+\|\boldsymbol{g}\|_{\Gamma}\right)
$$

for all $h \in\left(0, h_{0}\right]$.

Proof. Let $\left(\phi_{h}, \chi_{h}\right)$ be the solution to the discrete dual problem

$$
A_{h}\left((\boldsymbol{v}, q),\left(\phi_{h}, \chi_{h}\right)\right)=\left(\boldsymbol{\psi}^{e}, \boldsymbol{v}\right)_{\Gamma_{h}}
$$

where $\boldsymbol{\psi}: \Gamma \rightarrow \mathbb{R}^{3}$ is a given tangential vector field. We note that there is a unique solution to 5.99 , and using the technique in the proof of Lemma 5.2 , we conclude that the following stability estimate holds

$$
\left\|\left(\phi_{h}, \chi_{h}\right)\right\|_{h}+h^{-1}\left\|\boldsymbol{n} \cdot \boldsymbol{\phi}_{h}\right\|_{\Gamma_{h}} \lesssim\|\boldsymbol{\psi}\|_{\Gamma}
$$

Setting $(\boldsymbol{v}, q)=\left(\pi_{h} \boldsymbol{u}-\boldsymbol{u}_{h}, \pi_{h} p-p_{h}\right)$ in (5.99) we obtain the error representation formula

$$
\begin{aligned}
\left(\pi_{h} \boldsymbol{u}-\boldsymbol{u}_{h}, \boldsymbol{\psi}^{e}\right)_{\Gamma_{h}}= & A_{h}\left(\left(\pi_{h} \boldsymbol{u}-\boldsymbol{u}_{h}, \pi_{h} p-p_{h}\right),\left(\phi_{h}, \chi_{h}\right)\right) \\
= & A_{h}\left(\left(\pi_{h} \boldsymbol{u}, \pi_{h} p\right),\left(\phi_{h}, \chi_{h}\right)\right)-L_{h}\left(\left(\phi_{h}, \chi_{h}\right)\right) \\
= & A_{h}\left(\left(\pi_{h} \boldsymbol{u}, \pi_{h} p\right),\left(\phi_{h}, \chi_{h}\right)\right) \\
& \underbrace{-A\left((\boldsymbol{u}, p),\left(\phi_{h}, \chi_{h}\right)^{l}\right)+L\left(\left(\phi_{h}, \chi_{h}\right)^{l}\right)}_{=0}-L_{h}\left(\left(\boldsymbol{\phi}_{h}, \chi_{h}\right)\right) \\
= & A\left(\left(\pi_{h} \boldsymbol{u}, \pi_{h} p\right)^{l}-(\boldsymbol{u}, p),\left(\boldsymbol{\phi}_{h}, \chi_{h}\right)^{l}\right) \\
& \quad+A_{h}\left(\left(\pi_{h} \boldsymbol{u}, \pi_{h} p\right),\left(\phi_{h}, \chi_{h}\right)\right)-A\left(\left(\pi_{h} \boldsymbol{u}, \pi_{h} p\right)^{l},\left(\boldsymbol{\phi}_{h}, \chi_{h}\right)^{l}\right) \\
& +L\left(\left(\boldsymbol{\phi}_{h}, \chi_{h}\right)^{l}\right)-L_{h}\left(\left(\boldsymbol{\phi}_{h}, \chi_{h}\right)\right) \\
= & I I+I I+I I
\end{aligned}
$$

The terms may be estimated as follows.

Term I. We have

$$
\begin{aligned}
|I| & \lesssim\left\|\mid\left(\boldsymbol{u}-\left(\pi_{h} \boldsymbol{u}\right)^{l}, p-\left(\pi_{h} p\right)^{l}\right)\right\|\|\|\left(\boldsymbol{\phi}_{h}, \chi_{h}\right)\|\|_{h} \\
& \lesssim\left(h^{k_{u}+1}\|\boldsymbol{u}\|_{H^{k_{u}+1}(\Gamma)}+h^{k_{p}}\|p\|_{H^{k_{p}+1}(\Gamma)}\right)\|\boldsymbol{\psi}\|_{\Gamma}
\end{aligned}
$$

where we used continuity of $A_{h}$, the interpolation estimate 3.9 , and the stability estimate 5.100 .

Term II. We have

$$
\begin{aligned}
|I I| & \lesssim h^{k_{g}+1}\left(\|\|\left(\pi_{h} \boldsymbol{u}, \pi_{h} p\right)\|\|_{h}+h^{-1}\left\|\boldsymbol{n} \cdot \pi_{h} \boldsymbol{u}\right\|_{\Gamma_{h}}\right)\left(\left\|\left(\boldsymbol{\phi}_{h}, \chi_{h}\right)\right\|_{h}+h^{-1}\left\|\boldsymbol{n} \cdot \boldsymbol{\phi}_{h}\right\|_{\Gamma_{h}}\right) \\
& \lesssim h^{k_{g}+1}\left(\|\boldsymbol{u}\|_{H^{1}(\Gamma)}+\|p\|_{H^{1}(\Gamma)}\right) \boldsymbol{\psi} \|_{\Gamma}
\end{aligned}
$$

where we used the quadrature estimate (5.70), the stability of the interpolation operator, and the stability estimate 5.100 . 
Term III. We have

$$
\begin{aligned}
|I I I| & \lesssim h^{k_{g}+1}\left(\|f\|_{\Gamma}+\|\boldsymbol{g}\|_{\Gamma}\right)\left\|\left(\boldsymbol{\phi}_{h}, \chi_{h}\right)\right\|_{h} \\
& \lesssim h^{k_{g}+1}\left(\|f\|_{\Gamma}+\|\boldsymbol{g}\|_{\Gamma}\right)\|\boldsymbol{\psi}\|_{\Gamma}
\end{aligned}
$$

where we used the quadrature estimate (5.71) and the stability estimate 5.100).

Conclusion. Finally, setting $\boldsymbol{\psi}=\boldsymbol{P}_{\Gamma} \boldsymbol{e}_{h, u} /\left\|\boldsymbol{P}_{\Gamma} \boldsymbol{e}_{h, u}\right\|_{\Gamma}$ we have

$$
\left(\boldsymbol{e}_{h, u}, \boldsymbol{\psi}\right)_{\Gamma_{h}}=\left\|\boldsymbol{P}_{\Gamma} \boldsymbol{e}_{h, u}\right\|_{\Gamma_{h}} \sim\left\|\boldsymbol{P}_{\Gamma}\left(\boldsymbol{u}-\boldsymbol{u}_{h}^{l}\right)\right\|_{\Gamma_{h}}
$$

where we used equivalence of norms (4.19). Thus the proof is complete.

\section{Numerical Example: Flow on a Torus}

Let $\Gamma$ be the torus, given implicitly by the solution to

$$
\left(R-\sqrt{x^{2}+y^{2}}\right)^{2}+z^{2}-r^{2}=0
$$

were $R=1$ is the major and $r=1 / 2$ is the minor radius and let the right-hand side $\boldsymbol{g}$ correspond to the solution

$$
\boldsymbol{u}_{t}=\left(2 x z,-2 y z, 2\left(x^{2}-y^{2}\right)\left(R-\sqrt{x^{2}+y^{2}}\right) / \sqrt{x^{2}+y^{2}}\right), \quad u_{n}=0, \quad p=z .
$$

Note that $\operatorname{div}_{\Gamma} \boldsymbol{u}=0$, so $f=0$. The errors are computed on the discrete geometry by defining $e_{p}:=\left\|p^{e}-p_{h}\right\|_{\Gamma_{h}}$ and $e_{u}:=\left\|\boldsymbol{u}^{e}-\boldsymbol{u}_{h}\right\|_{\Gamma_{h}}$. For the evaluation of the integral $\left(\boldsymbol{g}_{h}, \boldsymbol{v}\right)_{\Gamma_{h}}$, we use $\boldsymbol{g}_{h}=\boldsymbol{g}^{e}$. We have used $c_{N}=0$, see Remark 3.1. We emphasize that $c_{N}>0$ does not affect the asymptotic convergence rate; however, for large $c_{N}$ a locking effect can occur. Moderate sizes of $c_{N}$ have a negligible effect on the error.

In Fig. 1 we show the computed velocity field on a particular mesh, with computed pressure isolevels on the same mesh in Fig. 2.

In order to make a comparison between structured and unstructured meshes, we create a sequence of unstructured meshes by randomly moving the nodes on each mesh in a sequence of structured meshes. A typical example is shown in Fig. 3. We then make eight comparisons:

We note the following: for Case 1 (Fig. 4), the geometry error does not affect the solution and we get optimal $O\left(h^{2}\right)$-convergence in pressure and superconvergence $O\left(h^{2}\right)$ convergence of velocities, related to the structuredness of the meshes. In Case 2 (Fig. 4), the increase in polynomial degree for the pressure does not help because of the poor geometry approximation and we keep $O\left(h^{2}\right)$-convergence.

For Case 3 (Fig. 5) we lose the superconvergence in velocity, which becomes $O(h)$; for Case 4 (Fig. 5) we regain optimal convergence for the velocity of $O\left(h^{2}\right)$ but, as for Case 2, 


\begin{tabular}{|ccccc|cc|}
\hline Case & $k_{u}$ & $k_{p}$ & $k_{g}$ & Mesh & Order $e_{u}$ & Order $e_{p}$ \\
\hline \hline 1 & 1 & 1 & 1 & Structured & 2 & 2 \\
\hline 2 & 1 & 2 & 1 & Structured & 2 & 2 \\
\hline 3 & 1 & 1 & 1 & Unstructured & 1 & 2 \\
\hline 4 & 1 & 2 & 1 & Unstructured & 2 & 2 \\
\hline 5 & 1 & 1 & 2 & Structured & 2 & 2 \\
\hline 6 & 1 & 2 & 2 & Structured & 2 & 3 \\
\hline 7 & 1 & 1 & 2 & Unstructured & 1 & 2 \\
\hline 8 & 1 & 2 & 2 & Unstructured & 2 & 3 \\
\hline
\end{tabular}

Table 1: The eight cases considered in the numerical examples together with the observed orders of convergence.

the geometry approximation precludes optimal convergence of the pressure which remains $O\left(h^{2}\right)$.

For Case 5 (Fig. 6) we have optimal convergence in pressure of $O\left(h^{2}\right)$ and superconvergence of velocity of $O\left(h^{2}\right)$. Improving the pressure approximation as in Case 6 (Fig. 6) now leads to the expected convergence of $O\left(h^{2}\right)$ for velocity and $O\left(h^{3}\right)$ for pressure.

On unstructured meshes, Case 7 (Fig. 7) we lose the superconvergence of velocities and obtain only $O(h)$ in velocity error with linear pressures, while Case 8 (Fig. 7) again gives the expected error of $O\left(h^{2}\right)$ for velocity and $O\left(h^{3}\right)$ for pressure.

We conclude that:

- Piecewise linear approximations for $\boldsymbol{u}_{h}$ and $p_{h}$ have superconvergence of velocity on structured meshes, both for piecewise linear and piecewise quadratic geometry.

- Increasing the polynomial degree of the pressure to $P^{2}$ increases the convergence of a piecewise linear velocity from $O(h)$ to $O\left(h^{2}\right)$ on unstructured meshes, both for piecewise linear and piecewise quadratic geometry.

- Increasing the convergence rate of the pressure, from $O\left(h^{2}\right)$ to $O\left(h^{3}\right)$, when going from $P^{1}$ to $P^{2}$ approximations requires that the same increase is being made in the geometry approximation.

Comments:

- For unstructured meshes these results are in accordance with the theoretical investigations in Section 5. We note, however, that in the numerical results the normal component of the error in the velocity also converges optimally, i.e. of order $k_{g}+1$, with respect to the order of approximation of the geometry while in Theorem 5.1 we achieve order $k_{g}$. In Theorem 5.3 we, however, show that the tangent component of the error is indeed optimal with respect to the order of the approximation of the geometry. Thus our theoretical results are in line with the numerical results but slightly weaker with respect to the order of approximation of the geometry for the 
normal component of the error. The error in the pressure and the tangent component are optimal with respect to $k_{p}, k_{u}$, and $k_{g}$.

- For structured meshes the superconvergence most certainly is related to superconvergence of $L^{2}$ projections of the gradient on the continuous space, see [1], which holds on structured meshes.

\section{References}

[1] R. E. Bank and J. Xu. Asymptotically exact a posteriori error estimators. I. Grids with superconvergence. SIAM J. Numer. Anal., 41(6):2294-2312 (electronic), 2003.

[2] S. C. Brenner and L. R. Scott. The Mathematical Theory of Finite Element Methods, volume 15 of Texts in Applied Mathematics. Springer, New York, third edition, 2008.

[3] E. Burman, P. Hansbo, and M. G. Larson. A stabilized cut finite element method for partial differential equations on surfaces: the Laplace-Beltrami operator. Comput. Methods Appl. Mech. Engrg., 285:188-207, 2015.

[4] A. Demlow. Higher-order finite element methods and pointwise error estimates for elliptic problems on surfaces. SIAM J. Numer. Anal., 47(2):805-827, 2009.

[5] G. Dziuk. Finite elements for the Beltrami operator on arbitrary surfaces. In Partial differential equations and calculus of variations, volume 1357 of Lecture Notes in Math., pages 142-155. Springer, Berlin, 1988.

[6] G. Dziuk and C. M. Elliott. Finite element methods for surface PDEs. Acta Numer., 22:289-396, 2013.

[7] A. Feronni, L. Formaggia, and A. Fumagalli. Numerical analysis of Darcy problem on surfaces. Technical Report 30, MOX, Dipartimento di Matematica "F. Brioschi" Politecnico di Milano, Via Bonardi 9 - 20133 Milano (Italy), 2014.

[8] D. Gilbarg and N. S. Trudinger. Elliptic Partial Differential Equations of Second Order. Classics in Mathematics. Springer-Verlag, Berlin, 2001. Reprint of the 1998 edition.

[9] P. Hansbo and M. G. Larson. Finite element modeling of a linear membrane shell problem using tangential differential calculus. Comput. Methods Appl. Mech. Engrg., 270:1-14, 2014.

[10] P. Hansbo, M. G. Larson, and F. Larsson. Tangential differential calculus and the finite element modeling of a large deformation elastic membrane problem. Comput. Mech., 56(1):87-95, 2015. 
[11] K. Larsson and M. G. Larson. A continuous/discontinuous Galerkin method and a priori error estimates for the biharmonic problem on surfaces. Technical report, Umeå University, Department of Mathematics, SE-90187, Umeå, Sweden, 2015. arXiv:1305.2740v2.

[12] A. Masud and T. J. R. Hughes. A stabilized mixed finite element method for Darcy flow. Comput. Methods Appl. Mech. Engrg., 191(39-40):4341-4370, 2002. 


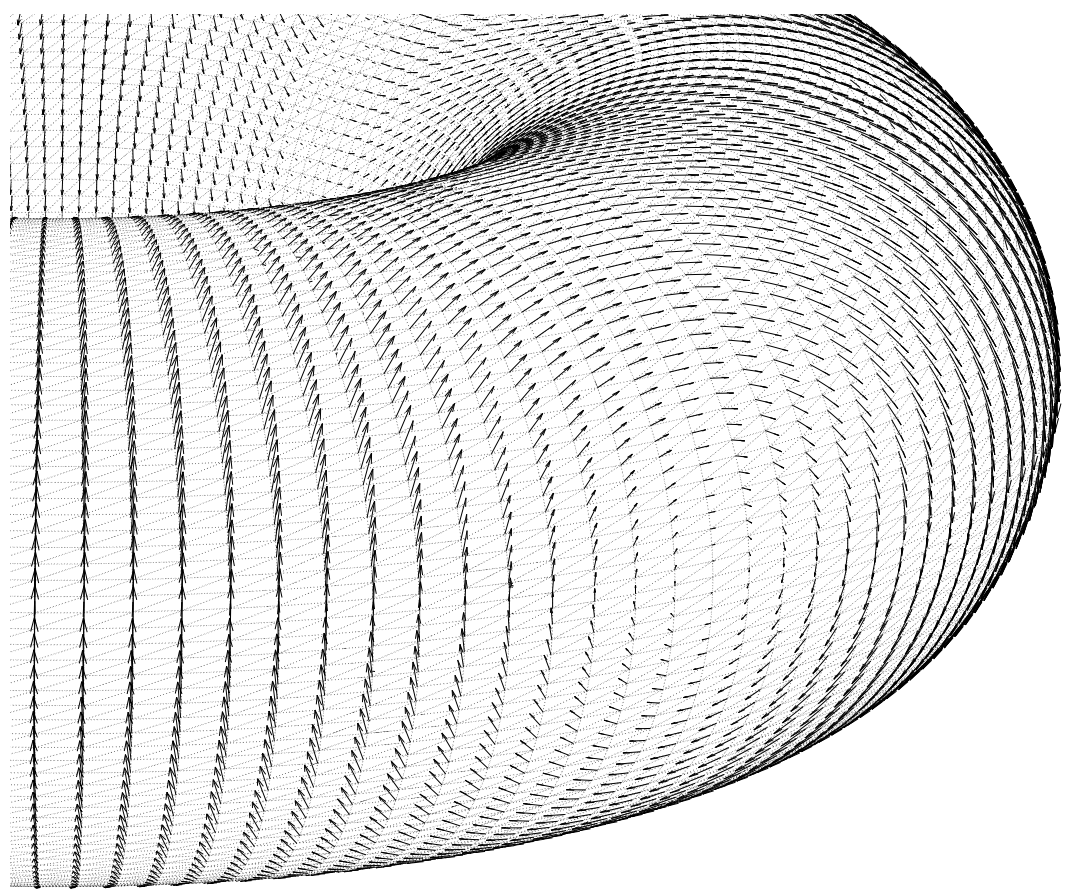

Figure 1: A detail of the computed velocity on a structured mesh. 


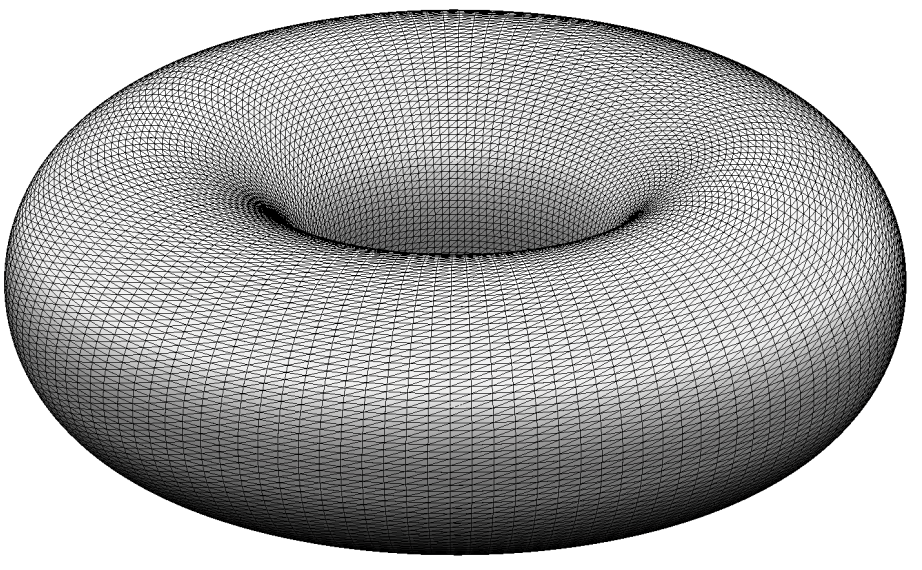

Figure 2: Computed pressure on a structured mesh. 


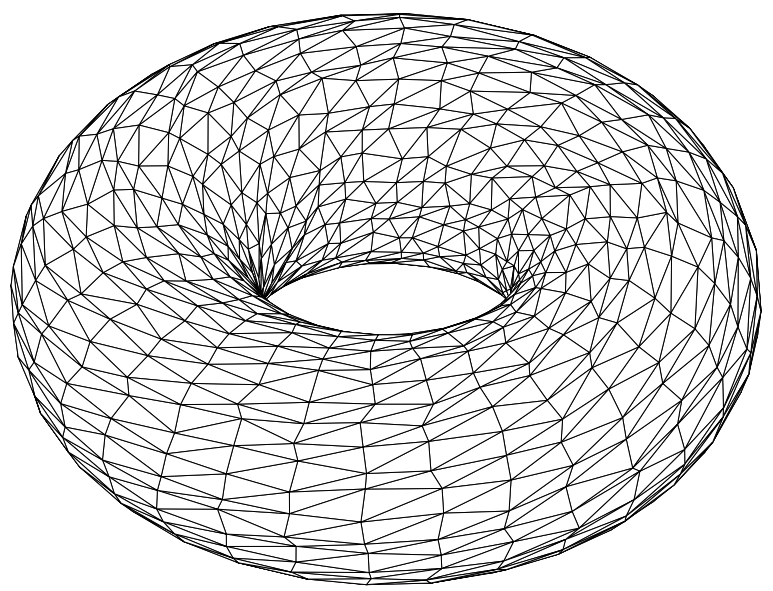

Figure 3: Unstructured mesh acquired by jiggling the nodes of a structured mesh. 


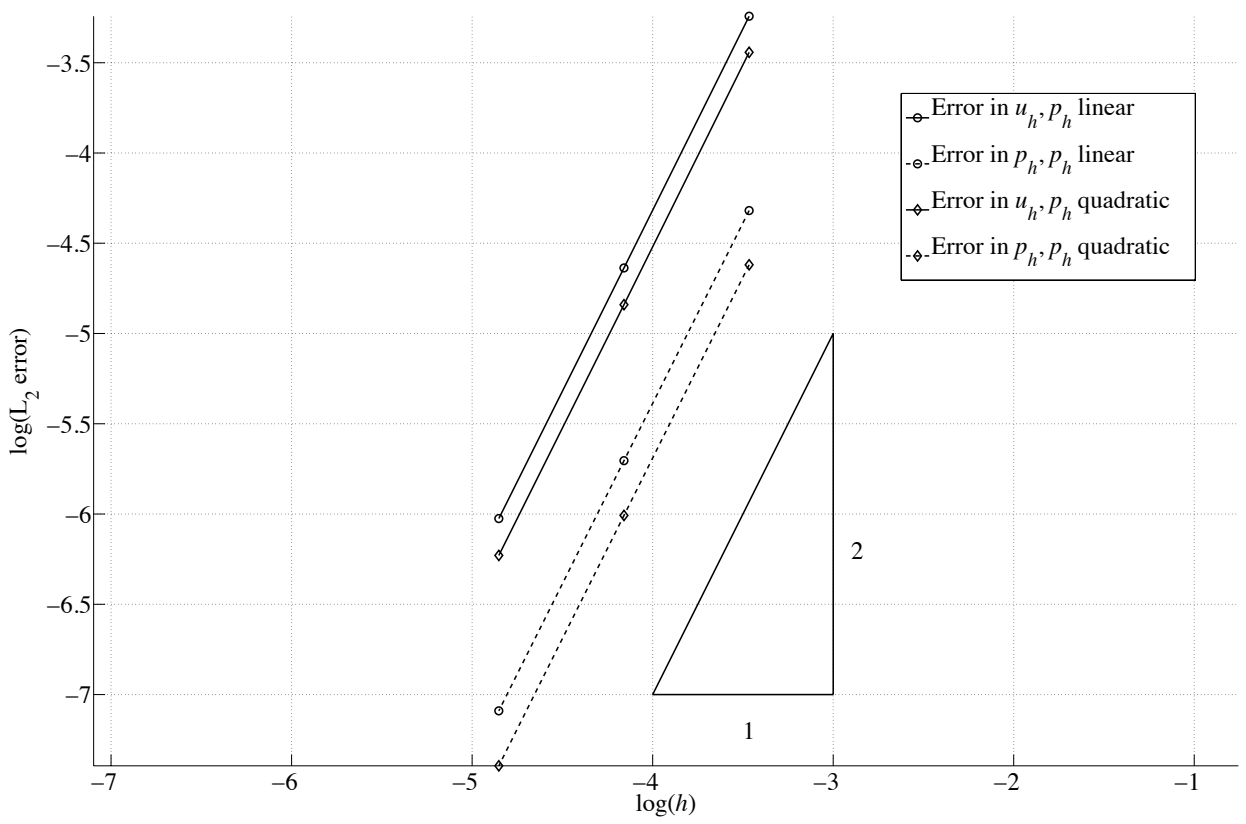

Figure 4: Computed errors on a piecewise linear, structured mesh. 


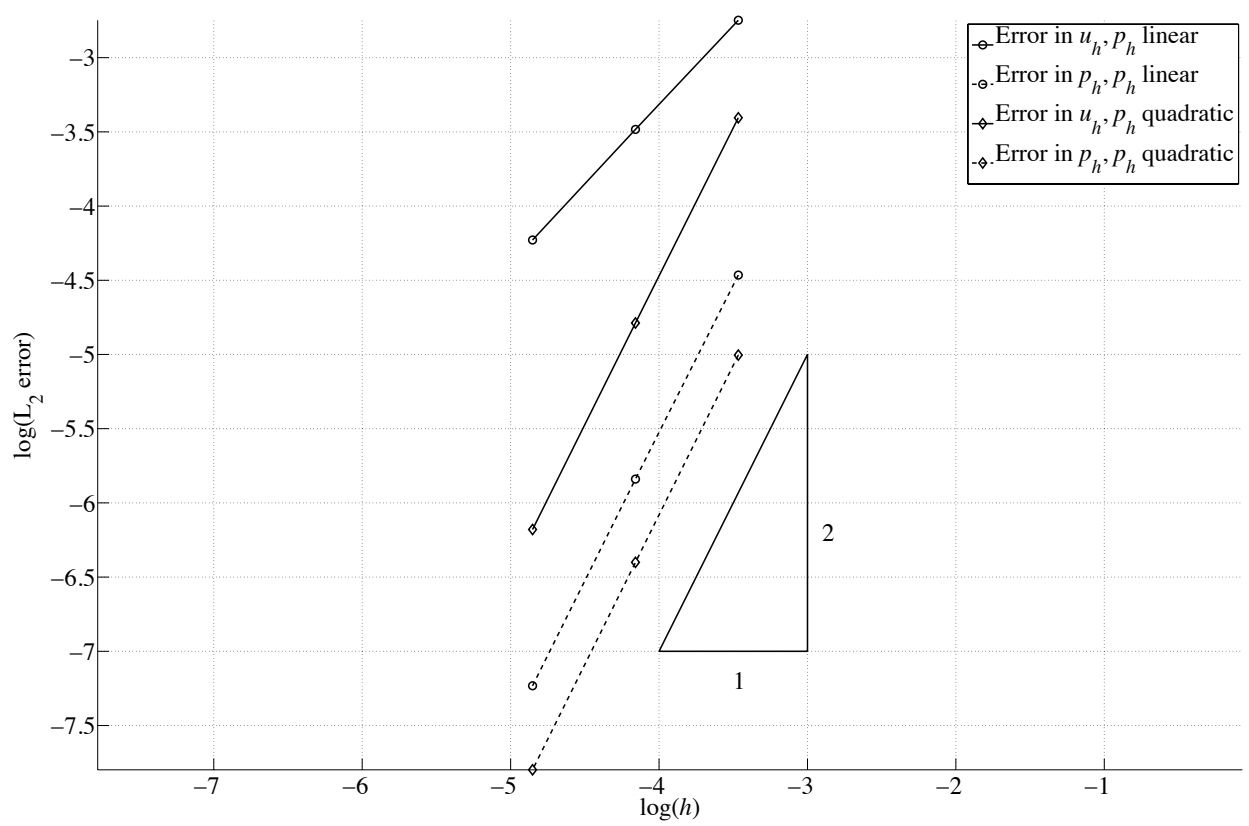

Figure 5: Computed errors on a piecewise linear, unstructured mesh. 


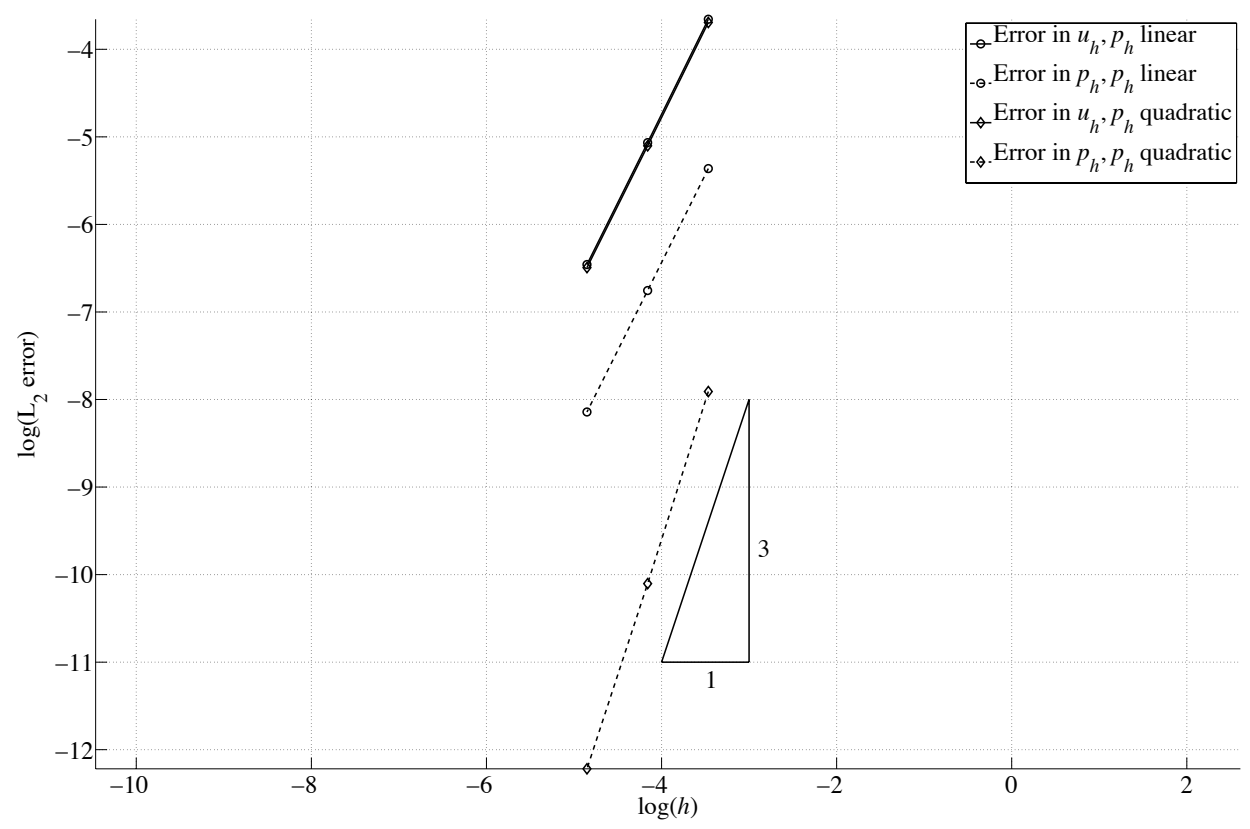

Figure 6: Computed errors on a piecewise quadratic, structured mesh. 


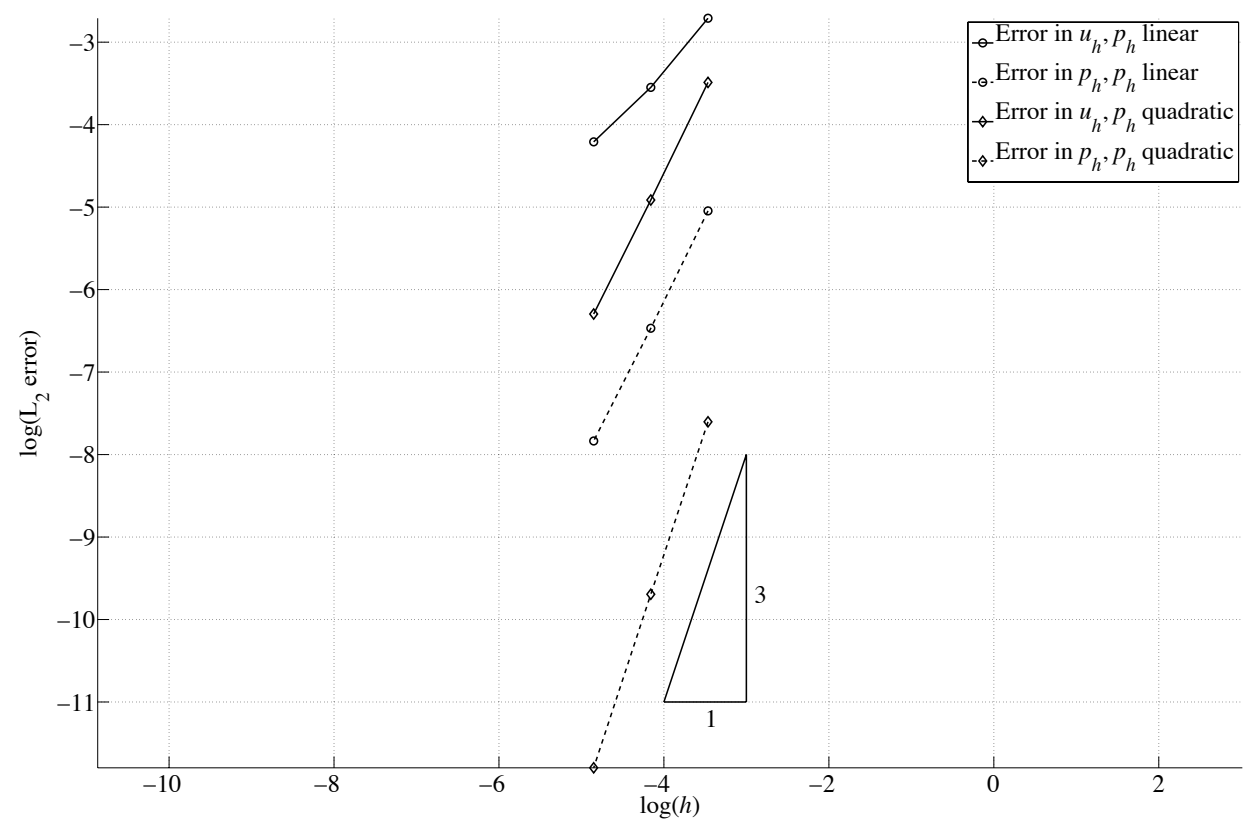

Figure 7: Computed errors on a piecewise quadratic, unstructured mesh. 